\title{
Finite and Infinitesimal Flexibility of Semidiscrete Surfaces
}

\author{
Oleg Karpenkov ${ }^{1}$
}

Received: 18 April 2015 / Revised: 28 July 2015 / Accepted: 24 August 2015 /

Published online: 3 September 2015

(C) Institute for Mathematical Sciences (IMS), Stony Brook University, NY 2015

\begin{abstract}
In this paper we study infinitesimal and finite flexibility for regular semidiscrete surfaces. We prove that regular 2-ribbon semidiscrete surfaces have one degree of infinitesimal and finite flexibility. In particular we write down a system of differential equations describing isometric deformations in the case of existence. Further we find a necessary condition of 3-ribbon infinitesimal flexibility. For an arbitrary $n \geq 3$ we prove that every regular $n$-ribbon surface has at most one degree of finite/infinitesimal flexibility. Finally, we discuss the relation between general semidiscrete surface flexibility and 3-ribbon subsurface flexibility. We conclude this paper with one surprising property of isometric deformations of developable semidiscrete surfaces.
\end{abstract}

Keywords Semidiscrete surfaces · Flexibility · Infinitesimal flexibility

\section{Introduction}

A mapping $f: \mathbb{R} \times \mathbb{Z} \rightarrow \mathbb{R}^{3}$, where the dependence on the continuous parameter is smooth, is called a semidiscrete surface. Let us connect $f(t, z)$ with $f(t, z+1)$ by segments for all possible pairs $(t, z)$. The resulting surface is a piecewise ruled surface.

In this paper we study infinitesimal and finite flexibility for such semidiscrete surfaces. By isometric deformations of a semidiscrete surface $f$ we understand deformations that preserve inner geometry of the corresponding ruled surfaces and in addition that preserve all line segments connecting $f(t, z)$ with $f(t, z+1)$.

The work is partially supported by FWF Grant No. S09209.

\footnotetext{
$\bowtie$ Oleg Karpenkov

O.Karpenkov@liverpool.ac.uk

1 University of Liverpool, Liverpool, UK
} 
Many questions on discrete polyhedral surfaces have their origins in the classical theory of smooth surfaces. Flexibility is no exception from this rule. The general theory of flexibility of surfaces and polyhedra is discussed in the overview by Sabitov (1992).

Bianchi (1890) introduced a necessary and sufficient condition for the existence of isometric deformations of a surface preserving some conjugate system (i.e., two independent smooth fields of directions tangent to the surface), see also in Eisenhart (1960), etc. Such surfaces can be understood as certain limits of semidiscrete surfaces.

On the other hand, semidiscrete surfaces are themselves the limits of certain polygonal surfaces (or meshes). For the discrete case of flexible meshes much is now known. Basing on paper by Stachel (2010) made a significant contribution to discrete case classifying all flexible Kokotsakis quadrilateral meshes (see Izmestiev 2014). We also refer the reader to Bobenko et al. (2008), Pottmann and Wallner (2008), Kokotsakis (1932), and Karpenkov (2010) for some other recent results in this area. For general relations to the classical case see a recent book by Bobenko and Suris (2008). It is interesting to notice that the necessary flexibility conditions in the smooth case and the discrete case are of a different nature. Currently there is no clear description of relations between them in terms of limits.

The place of the study of semidiscrete surfaces is between the classical and the discrete cases. Main concepts of semidiscrete theory are described by Wallner (2009, 2012). Some problems related to isothermic semidiscrete surfaces are studied by Müller and Wallner (2013). Semidiscrete surfaces from the viewpoint of parallelity, offsets, and curvatures were studied by Karpenkov and Wallner (2014).

We investigate necessary condition for existence of isometric deformations of semidiscrete surfaces. To avoid pathological behavior related to noncompactness of semidiscrete surfaces we restrict ourselves to compact subsets of the following type. An $n$-ribbon surface is a mapping

$$
f:[a, b] \times\{0, \ldots, n\} \rightarrow \mathbb{R}^{3}, \quad(t, i) \mapsto f_{i}(t)
$$

We also use the notion

$$
\Delta f_{i}(t)=f_{i+1}(t)-f_{i}(t)
$$

While working with a rather abstract semidiscrete or $n$-ribbon surface $f$ we keep in mind the two-dimensional piecewise-ruled surface associated to it (see Fig. 1).

Note that, within this paper we traditionally consider $t$ as an argument of a semidiscrete surface $f$. The time parameter for deformations is $\lambda$.

In present paper we prove that every regular 2-ribbon surface (as a ruled surface) is flexible and has one degree of infinitesimal and finite flexibility in the regular case (Theorems 1,2). This is quite surprising since regular 1-ribbon surfaces have infinitely many degrees of flexibility, see, for instance, in Pottmann and Wallner (2001, Theorem 5.3.10). We also find a system of differential equations for the deformation of 2-ribbon surfaces (Definition 18 and Proposition 9). In contrast to that, a regular $n$ ribbon surface is rigid for $n \geq 3$. For the case $n=3$ we prove the following statement (see Theorem 3 and Remark 10). 


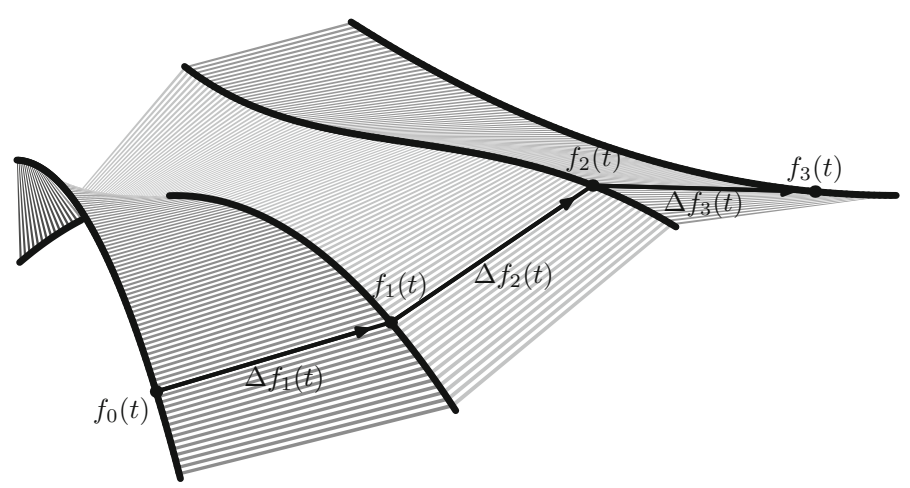

Fig. 1 A 3-ribbon surface

\subsection{Infinitesimal Flexibility Condition}

If a 3-ribbon surface is infinitesimally flexible then the following condition holds:

$$
\dot{\Lambda}=\left(H_{2}-H_{1}\right) \Lambda
$$

where

$$
\Lambda=\frac{\left(\dot{f}_{1}, \ddot{f}_{1}, \Delta f_{0}\right)}{\left(\dot{f}_{2}, \ddot{f}_{2}, \Delta f_{2}\right)} \frac{\left(\dot{f}_{2}, \Delta f_{1}, \Delta f_{2}\right)^{2}}{\left(\dot{f}_{1}, \Delta f_{0}, \Delta f_{1}\right)^{2}}
$$

and

$$
H_{i}(t)=\frac{\left(\dot{f}_{i}, \Delta \dot{f}_{i-1}, \Delta f_{i}\right)+\left(\dot{f}_{i}, \Delta f_{i-1}, \Delta \dot{f}_{i}\right)}{\left(\dot{f}_{i}, \Delta f_{i-1}, \Delta f_{i}\right)}, \quad i=1,2 .
$$

Remark Throughout this paper we denote the derivative with respect to variable $t$ by the dot symbol.

Further in Theorem 4 we state that a regular $n$-ribbon surface $(n \geq 3)$ has at most one degree of finite and infinitesimal flexibility. Finally, we show that a regular $n$-ribbon surface $(n \geq 4)$ is infinitesimally or finitely flexible if and only if all its 3 -ribbon subsurfaces are infinitesimally or finitely flexible (see Theorems 7 and 8). We say a few words in the case of developable semidiscrete surfaces whose finite isometric deformations have additional surprising properties. Let us mention that a similar flexibility question for smooth conjugate nets was solved by Eisenhart (1960) (see Section 141).

\subsection{Organization of the Paper}

We start in Sect. 2 with the introduction of necessary notions and definitions. In Sect. 3 we discuss flexibility of 2-ribbon surfaces. We study infinitesimal flexibility questions 
for 2-ribbon surfaces in Sects. 3.2 and 3.3. In Sect. 3.2 we give a system of differential equations for infinitesimal flexions, prove the existence of nonzero solutions, and show that all the solutions are proportional to each other. In Sect. 3.3 we define the operators of infinitesimal flexion which is studied further in the context of finite flexibility for 2-ribbon surfaces. In Sect. 3.4 we prove that a regular 2-ribbon surface is finitely flexible and has one degree of flexibility. In Sect. 4 we work with 3-ribbon surfaces. After some preliminary statements of Sect. 4.1 we gives a necessary infinitesimal flexibility condition for 3-ribbon surfaces in Sect. 4.2. In Sect. 5 we deal with general $n$-ribbon surfaces for $n \geq 3$. We prove that a regular $n$-ribbon surface has at most one degree of finite and infinitesimal flexibility in Sect. 5.1. Further after several preparatory statements of Sect. 5.2 we prove that finite or infinitesimal flexibility of regular $n$-ribbon surfaces is identified by finite or infinitesimal flexibility of all its 3 ribbon subsurfaces. We conclude the paper with flexibility of developable semidiscrete surfaces in Sect. 6. In this case isometric deformations have a remarkable geometric property.

\section{Necessary Notions and Definitions}

In this section we introduce central notions and definition of the article.

\subsection{Differentiable Regular Semidiscrete Surfaces}

We start with several basic definitions.

Definition 1 Let $M=\left(m_{0}, \ldots, m_{n}\right)$ be the $(n+1)$-tuple of non-negative integers. We say that an $n$-ribbon surface $f$ is a $M$-differentiable if for every $i \in\{0, \ldots, n\}$ and $j \in\left\{1, \ldots, m_{i}\right\}$ there exists a continuous derivative $f_{i}^{(j)}$.

Denote by $C^{m_{0}, \ldots, m_{n}}\left([a, b], \mathbb{R}^{3}\right)$ (or $C^{M}\left([a, b], \mathbb{R}^{3}\right)$, for short) the Banach space of all $M$-differentiable $n$-ribbon surfaces (where $t \in[a, b]$ ) with the standard norm

$$
\rho(f, g)=\max _{i=\{0, \ldots, n\}} \max _{j=\left\{1, \ldots, m_{i}\right\}} \sup _{[a, b]}\left(f_{i}^{(j)}-g_{i}^{(j)}\right) .
$$

Remark 1 Note that for two non-negative $(n+1)$-tuples $M=\left(m_{0}, \ldots, m_{n}\right)$ and $K=$ $\left(k_{0}, \ldots, k_{n}\right)$ satisfying

$$
m_{0} \geq k_{0}, \quad \ldots, \quad m_{n} \geq k_{n}
$$

we have

$$
C^{M}\left([a, b], \mathbb{R}^{3}\right) \subset C^{K}\left([a, b], \mathbb{R}^{3}\right) .
$$

Definition 2 We say that an $n$-ribbon surface $f \in C^{1,2,2, \ldots, 2,1}\left([a, b], \mathbb{R}^{3}\right)$ is weakly regular if for every $t \in[a, b]$ and $i=1, \ldots, n-1$ we have

$$
\left(\dot{f}_{i}, \Delta f_{i-1}, \Delta f_{i}\right) \neq 0 \text {. }
$$


Definition 3 We say that an $n$-ribbon surface $f \in C^{1,2,2, \ldots, 2,1}\left([a, b], \mathbb{R}^{3}\right)$ is strongly regular if

- $f$ is weakly regular;

- for every $t \in[a, b]$ and $i=1, \ldots, n-1$ we have

$$
\left(\dot{f}_{i}(t), \ddot{f}_{i}(t), \Delta f_{i-1}(t)\right) \neq 0 \quad \text { and } \quad\left(\dot{f}_{i}(t), \ddot{f}_{i}(t), \Delta f_{i}(t)\right) \neq 0
$$

\subsection{Isometric Semidiscrete Surfaces}

Let us now study basic properties of the definition of isometric semidiscrete surfaces.

Definition 4 Two $n$-ribbon surfaces $f$ and $g$ in the space $C^{1,1, \ldots, 1}\left([a, b], \mathbb{R}^{3}\right)$ are said to be isometric if

$$
\left\{\begin{array}{l}
\left|\dot{f}_{i}\right|=\left|\dot{g}_{i}\right| \\
\left|\Delta f_{i}\right|=\left|\Delta g_{i}\right| \\
\left\langle\dot{f}_{i}, \Delta f_{i-1}\right\rangle=\left\langle\dot{g}_{i}, \Delta g_{i-1}\right\rangle \\
\left\langle\dot{f}_{i}, \Delta f_{i}\right\rangle=\left\langle\dot{g}_{i}, \Delta g_{i}\right\rangle \\
\left\langle\dot{f}_{i}, \dot{f}_{i+1}\right\rangle=\left\langle\dot{g}_{i}, \dot{g}_{i+1}\right\rangle
\end{array}\right.
$$

(for all admissible $i$ and $t$ ).

Before we continue let us show that the conditions of Definition 4 are precisely the isometric conditions for ruled surfaces. Let $f_{1}$ and $f_{2}$ be differentiable curves (denote by $\Delta_{1} f$ the curve $\left.f_{2}-f_{1}\right)$. Let us define a ruled surface $S(x, t)=x f_{1}(t)+(1-x) f_{2}(t)$. To show that the conditions of Definition 4 determine inner geometry we prove the following proposition.

Proposition 1 The first fundamental form of the ruled surface $S(x, t)$ is uniquely defined by

$$
\left|\dot{f}_{1}\right|, \quad\left|\dot{f}_{2}\right|, \quad\left|\Delta f_{1}\right|, \quad\left\langle\dot{f}_{1}, \Delta f_{1}\right\rangle, \quad\left\langle\dot{f}_{2}, \Delta f_{1}\right\rangle, \quad\left\langle\dot{f}_{1}, \dot{f}_{2}\right\rangle
$$

and vice versa.

Proof Let us write all the coefficients of the first fundamental form of the surface in the coordinates $(x, t)$ :

$$
\begin{aligned}
\left\langle\frac{\partial S}{\partial x}, \frac{\partial S}{\partial x}\right\rangle & =\left\langle f_{1}-f_{2}, f_{1}-f_{2}\right\rangle=\left|\Delta f_{1}\right|^{2} \\
\left\langle\frac{\partial S}{\partial x}, \frac{\partial S}{\partial t}\right\rangle & =\left\langle f_{1}-f_{2}, x \dot{f}_{1}+(1-x) \dot{f}_{2}\right\rangle=x\left\langle\Delta f_{1}, \dot{f}_{1}\right\rangle+(1-x)\left\langle\Delta f_{1}, \dot{f}_{2}\right\rangle \\
\left\langle\frac{\partial S}{\partial t}, \frac{\partial S}{\partial t}\right\rangle & =\left\langle x \dot{f}_{1}+(1-x) \dot{f}_{2}(t), x \dot{f}_{1}+(1-x) \dot{f}_{2}(t)\right\rangle \\
& =x^{2}\left|f_{1}\right|^{2}+2 x(1-x)\left\langle\dot{f}_{1}, \dot{f}_{2}\right\rangle+(1-x)^{2}\left|f_{2}\right|^{2}
\end{aligned}
$$


As we see, on the one hand the first fundamental form is defined by the above six functions. On the other hand the values of the first fundamental form at $x=0,1 / 2,1$ defines the values of the above six functions.

\subsection{Deformations and Flexions of Semidiscrete Surfaces}

We start with the following general definition.

Definition 5 A deformation of a semidiscrete $n$-ribbon surface $f$ is a family of $n$ ribbon surfaces $\left\{f^{\lambda}\right\}$ with parameter $\lambda$ in the interval $[-\Lambda, \Lambda]$ for some positive $\Lambda$ such that $f^{0}=f$. In this paper we consider only deformations that are continuously differentiable in $\lambda$.

Remark 2 In this paper $\lambda$ is the parameter of deformations, while $t$ is the first argument of semidiscrete surfaces.

Let us give a formal definition of deformations that do not change the inner geometry of a surface.

Definition 6 We say that a deformation $\left\{f^{\lambda}\right\}$ of a semidiscrete $n$-ribbon surface $f$ is isometric if all the surfaces in the deformation are isometric to each other.

Definition 7 Consider a family of functions, vector functions, or semidiscrete surfaces $\gamma=\left\{w^{\lambda}\right\}$ with parameter $\lambda \in[-\varepsilon, \varepsilon]$ for some positive $\varepsilon$, and let $w=w^{0}$. We say that the derivative

$$
\mathcal{D}_{\gamma} w=\left.\frac{\partial w^{\lambda}}{\partial \lambda}\right|_{\lambda=0}
$$

is an infinitesimal deformation of $w$.

The infinitesimal deformation of an $n$-ribbon surface $f$ in $C^{M}\left([a, b], \mathbb{R}^{3}\right)$ is an element of the tangent space $T_{f} C^{M}\left([a, b], \mathbb{R}^{3}\right)$, which is naturally isomorphic to $C^{M}\left([a, b], \mathbb{R}^{3}\right)$.

Definition 8 Consider a deformation $\left\{f^{\lambda}\right\}$ of a semidiscrete $n$-ribbon surface $f$ in $C^{(1,2,2, \ldots, 2,1)}\left([a, b], \mathbb{R}^{3}\right)$. We say that the deformation $\left\{f^{\lambda}\right\}$ is infinitesimally flexible if

$$
\begin{gathered}
\mathcal{D}_{\gamma}\left|\dot{f}_{i}^{\lambda}\right|=0, \quad \mathcal{D}_{\gamma}\left|\Delta f_{i}^{\lambda}\right|=0, \quad \mathcal{D}_{\gamma}\left\langle\dot{f}_{i}^{\lambda}, \Delta f_{i-1}^{\lambda}\right\rangle=0, \\
\mathcal{D}_{\gamma}\left\langle\dot{f}_{i}^{\lambda}, \Delta f_{i}^{\lambda}\right\rangle=0, \quad \text { and } \mathcal{D}_{\gamma}\left\langle\dot{f}_{i}^{\lambda}, \dot{f}_{i+1}^{\lambda}\right\rangle=0
\end{gathered}
$$

(for all admissible $i$ and $t$ ).

In fact, infinitesimal flexibility is a property of tangent spaces rather than deformations. 
Definition 9 We say that a tangent vector $\mathcal{D} f$ at a semidiscrete surface $f$ is an infinitesimal flexion if the deformation $\mathcal{D}_{\gamma} f$ where

$$
\gamma(\lambda)=f+\lambda \mathcal{D} f
$$

is infinitesimally isometric.

We say that an infinitesimal flexion $\mathcal{D} f$ is a finite flexion if there exists an isometric deformation $\gamma$ with $\gamma(0)=f$ such that $\mathcal{D}_{\gamma} f=\mathcal{D} f$.

Finally let us determine isometrically nontrivial infinitesimal flexions.

Definition 10 An infinitesimal flexion of a weakly regular $n$-ribbon surface $f$ in $C^{0,1,0}\left([a, b], \mathbb{R}^{3}\right)$ is said to be isometrically nontrivial (trivial) at point $(t, i)$ for some $t \in[a, b]$ and $n \in\{1, \ldots, n-1\}$ if the corresponding infinitesimal deformation of the angle between the planes spanned by $\left(\dot{f}_{i}(t) \Delta f_{i-1}(t)\right)$ and $\left(\dot{f}_{i}(t) \Delta f_{i}(t)\right)$ is nonzero (or zero, respectively).

We say that an infinitesimal flexion of $f$ is isometrically nontrivial if it is isometrically nontrivial at least at one point $(t, i)$. Otherwise an infinitesimal inflexion is said to be isometrically trivial.

We say that an infinitesimal flexion of $f$ is strongly isometrically nontrivial if it is isometrically nontrivial at every point $(t, i)$.

\subsection{Spaces of Semidiscrete Surfaces with Fixed Initial Position}

In order to calculate the degree of flexibility for a semidiscrete surface we should eliminate trivial Euclidean deformations of the surfaces. Let us do this as follows.

Definition 11 Denote by

$$
C_{0}^{M}\left([a, b], \mathbb{R}^{3}\right) \subset C^{M}\left([a, b], \mathbb{R}^{3}\right)
$$

the subset of all 2-ribbon surfaces with fixed initial position, namely an $n$-ribbon surface $f$ is in $C_{0}^{M}\left([a, b], \mathbb{R}^{3}\right)$ if and only if

$-f_{1}(0) \in C^{M}\left([a, b], \mathbb{R}^{3}\right)$;

$-f_{1}(0)=(0,0,0)$

- the vector $\dot{f}_{1}(0)$ is proportional to $(1,0,0)$;

- the vector $\Delta f_{0}(0)$ has the coordinates $(p, q, 0)$.

Remark 3 Let $\Sigma$ denote all weakly non-regular semidiscrete surfaces. Notice that the set $C_{0}^{M}\left([a, b], \mathbb{R}^{3}\right) \backslash \Sigma$ has a natural structure of an 8 -fold covering of the quotient space of $C^{M}\left([a, b], \mathbb{R}^{3}\right) \backslash \Sigma$ by the Euclidean congruence relation. In other words, for every weakly regular $M$-differentiable semidiscrete surface $f$ there exists exactly eight semidiscrete surfaces that are congruent to $f$. These 8 surfaces are obtained one from another by 8 symmetries of type

$$
\left(e_{1}, e_{2}, e_{3}\right) \rightarrow\left( \pm e_{1}, \pm e_{2}, \pm e_{3}\right) .
$$


So, on the one hand one can consider any branch of the 8-fold for studying flexibility properties of the original $n$-ribbon curve. On the other hand the set $C_{0}^{M}\left([a, b], \mathbb{R}^{3}\right)$ has a structure of a vector space. For these reasons from now on we prefer to consider the space $C_{0}^{M}\left([a, b], \mathbb{R}^{3}\right)$, rather than the quotient space of $C^{M}\left([a, b], \mathbb{R}^{3}\right) \backslash \Sigma$ by the group of all Euclidean transformation.

Since $C_{0}^{M}\left([a, b], \mathbb{R}^{3}\right)$ is a subspace of $C^{M}\left([a, b], \mathbb{R}^{3}\right)$ we have the induced metric and topology (in particular, $C_{0}^{M}\left([a, b], \mathbb{R}^{3}\right)$ is a Banach space), definitions of deformations, isometric deformations, infinitesimal and finite flexions, isometrically trivial and nontrivial infinitesimal flexions in $C_{0}^{M}\left([a, b], \mathbb{R}^{3}\right)$.

\subsection{Rigid Semidiscrete Surfaces: Degrees of Flexibility}

We start with the definitions for infinitesimal flexibility.

Definition 12 The set of infinitesimal flexions in $C_{0}^{M}\left([a, b], \mathbb{R}^{3}\right)$ is a linear space. We say that $f$ has $n$ degrees of infinitesimal flexibility if the dimension of the space of infinitesimal flexions is $n$. If $n=0$ we say that $f$ is infinitesimally rigid.

In the finite case we define only finitely regularly rigid semidiscrete surfaces and surfaces that has one degree of finite flexibility. In order to define finite regular rigidity we use the following definition.

Definition 13 We say that an isometric deformation $\gamma$ of $f$ in $C_{0}^{M}\left([a, b], \mathbb{R}^{3}\right)$ is regular at 0 if $\mathcal{D}_{\gamma} f \neq 0$.

Definition 14 We say that an $n$-ribbon surface $f$ in $C_{0}^{M}\left([a, b], \mathbb{R}^{3}\right)$ is finitely regularly rigid if the set of regular isometric deformations of $f$ is empty.

Let us finally give the definition of the property to have one degree of finite flexibility. As in infinitesimal case we consider only the space of semidiscrete surfaces with fixed initial position $C_{0}^{M}\left([a, b], \mathbb{R}^{3}\right)$. This cancels excess trivial Euclidean rotations of the whole semidiscrete surface. Of course, every finite isometric deformations of a semidiscrete surface with fixed initial position still can be reparametrised, as a result one has another isometric deformation of the surface. So the best thing would be to try to normalize them.

In this paper we consider the following "natural parametrization" of an isometric deformation. It is clear that for every isometric deformation $\left\{f^{\lambda}\right\}$ in $C_{0}^{M}\left([a, b], \mathbb{R}^{3}\right)$ we have

$$
\mathcal{D}_{f^{\lambda}} \dot{f}(a)=0, \quad \mathcal{D}_{f^{\lambda}} \Delta f_{0}(a)=0, \quad \text { and } \quad \mathcal{D}_{f^{\lambda}} \Delta f_{1}(a)=\alpha(\lambda) \dot{f}(a) \times \Delta f_{1}(a)
$$

for some real valued function $\alpha$.

Definition 15 We say that an isometric deformation $\left\{f^{\lambda}\right\}$ is normalized if and only if for every admissible values of parameter $\lambda$ we have $\alpha(\lambda)=1$, where $\alpha$ is the real-valued function defined in the last expression. 
In our case by Corollary 1 below we have: if $\alpha\left(\lambda_{0}\right)=0$ then $\mathcal{D}_{f^{\lambda}} f^{\lambda_{0}}=$ 0 . Hence, there is no regular isometric deformation that preserves the frame ( $\left.\dot{f}_{1}(a), \Delta f_{0}(a), \Delta f_{1}(a)\right)$ and the point $f_{1}(a)$. So we can give the following definition.

Definition 16 We say that a weakly regular 2-ribbon surface $f$ has one degree of finite flexibility if

- $f$ has one degree of infinitesimal flexibility.

- for sufficiently small $\varepsilon>0$ there exists a unique normalized isometric deformation of $f$ defined on $[-\varepsilon, \varepsilon]$.

\section{Finite and Infinitesimal Flexibility of 2-Ribbon Surfaces}

In this section we describe flexions of 2-ribbon surfaces. Such surfaces are defined by three curves $f_{0}, f_{1}$, and $f_{2}$. Our main goal here is to prove under some natural genericity assumptions that every 2-ribbon surface is infinitesimally and finitely flexible and has one degree of infinitesimal and finite flexibility. Our first point is to describe the system of differential equations (System A) that determines infinitesimal flexions corresponding to finite flexions and find solutions to this system (see Sects. 3.2). We use it to derive finite flexibility in Theorem 1 (also in Sect. 3.2). Further via solutions of System A we define the operators of infinitesimal flexion $\mathcal{V}^{ \pm}$(in Sect. 3.3). Finally, to show finite flexibility of 2-ribbon surfaces we study Lipschitz properties for $\mathcal{V}^{ \pm}$ and prove flexibility Theorem 2 (in Sect. 3.4).

\subsection{Basic Relations for Infinitesimal Flexions}

In this small subsection we collect some useful relations.

Proposition 2 Let $f$ be a 2-ribbon surface in $C^{1,2,1}\left([a, b], \mathbb{R}^{3}\right)$. Then for every infinitesimal flexion $\mathcal{D} f$ the following properties hold:

$$
\begin{aligned}
& \left\langle\dot{f}_{1}, \mathcal{D} \dot{f}_{1}\right\rangle=0 ; \\
& \left\langle\dot{f}_{1}-\Delta \dot{f}_{0}, \mathcal{D} \dot{f}_{1}-\mathcal{D} \Delta \dot{f}_{0}\right\rangle=0 ; \\
& \left\langle\dot{f}_{1}+\Delta \dot{f}_{1}, \mathcal{D} \dot{f}_{1}+\mathcal{D} \Delta \dot{f}_{1}\right\rangle=0 ; \\
& \left\langle\Delta f_{0}, \mathcal{D} \Delta \dot{f}_{0}\right\rangle+\left\langle\Delta \dot{f}_{0}, \mathcal{D} \Delta f_{0}\right\rangle=0 ; \\
& \left\langle\Delta f_{1}, \mathcal{D} \Delta \dot{f}_{1}\right\rangle+\left\langle\Delta \dot{f}_{1}, \mathcal{D} \Delta f_{1}\right\rangle=0 ; \\
& \left\langle\dot{f}_{1}, \mathcal{D} \Delta \dot{f}_{0}\right\rangle+\left\langle\mathcal{D} \dot{f}_{1}, \Delta \dot{f}_{0}\right\rangle=0 ; \\
& \left\langle\dot{f}_{1}, \mathcal{D} \Delta \dot{f}_{1}\right\rangle+\left\langle\mathcal{D} \dot{f}_{1}, \Delta \dot{f}_{1}\right\rangle=0 ; \\
& \left\langle\mathcal{D} \ddot{f}_{1}, \Delta f_{0}\right\rangle+\left\langle\ddot{f}_{1}, \mathcal{D} \Delta f_{0}\right\rangle=0 ; \\
& \left\langle\mathcal{D} \ddot{f}_{1}, \Delta f_{1}\right\rangle+\left\langle\ddot{f}_{1}, \mathcal{D} \Delta f_{1}\right\rangle=0 .
\end{aligned}
$$

Remark 4 For a semidiscrete or $n$-ribbon surface $f$ the operations $\mathcal{D}, \Delta$, and $\frac{\partial}{\partial t}$ commute, so we do not pay attention to the order of these operations in compositions. 
Proof Equations (1), (2), and (3) follow from the fact that infinitesimal flexions preserve the norms of $\dot{f}_{1}, \dot{f}_{0}=\dot{f}_{1}-\Delta \dot{f}_{0}$, and $\dot{f}_{2}=\dot{f}_{1}+\Delta \dot{f}_{1}$ respectively.

The invariance of the lengths of $\Delta f_{0}$ and $\Delta f_{1}$ imply Equations (4), and (5) respectively. They are equivalent to

$$
\frac{\partial}{\partial t} \mathcal{D}\left\langle\Delta f_{0}, \Delta f_{0}\right\rangle=0 \text { and } \frac{\partial}{\partial t} \mathcal{D}\left\langle\Delta f_{1}, \Delta f_{1}\right\rangle=0
$$

Equations (6) and (7) follow from invariance of the angles between the vectors $\dot{f}_{1}$ and $\Delta \dot{f}_{0}$ and the vectors $\dot{f}_{1}$ and $\Delta \dot{f}_{0}$.

Let us prove Eq. (8). Since the angles between the vectors $\Delta f_{0}$ and $\dot{f}_{1}$ are preserved by infinitesimal flexions we have

$$
\frac{\partial}{\partial t} \mathcal{D}\left\langle\dot{f}_{1}, \Delta f_{0}\right\rangle=0
$$

Therefore,

$$
\left\langle\mathcal{D} \ddot{f}_{1}, \Delta f_{0}\right\rangle+\left\langle\ddot{f}_{1}, \mathcal{D} \Delta f_{0}\right\rangle+\left\langle\mathcal{D} \dot{f}_{1}, \Delta \dot{f}_{0}\right\rangle+\left\langle\dot{f}_{1}, \mathcal{D} \Delta \dot{f}_{0}\right\rangle=0
$$

By Eq. (6) we have $\left\langle\mathcal{D} \dot{f}_{1}, \Delta \dot{f}_{0}\right\rangle+\left\langle\dot{f}_{1}, \mathcal{D} \Delta \dot{f}_{0}\right\rangle=0$ and hence

$$
\left\langle\mathcal{D} \ddot{f}_{1}, \Delta f_{0}\right\rangle+\left\langle\ddot{f}_{1}, \mathcal{D} \Delta f_{0}\right\rangle=0
$$

We have arrived at Eq. (8).

Finally Eq. (9) is proved by analogy with Eq. (8).

\subsection{Infinitesimal Flexibility of 2-Ribbon Surfaces}

Our main goal for this subsection is to prove the following general theorem

Theorem 1 Let $f \in C_{0}^{1,2,1}\left([a, b], \mathbb{R}^{3}\right)$ be a weakly regular 2-ribbon surface with fixed initial position. Then $f$ has one degree of infinitesimal flexibility.

First we write down and investigate a supplementary system of differential equations (System A) which describes infinitesimal flexions of weakly regular 2-ribbon surfaces. We also show the uniqueness of the solution of System A for a given initial data (Proposition 3). The remaining part of this subsection is dedicated to the proof of Theorem 1 mentioned above. In Proposition 4 we show that every infinitesimal flexion satisfies System A. Then in Proposition 5 we prove that every solution of System A with certain initial data is an infinitesimal flexion. After that we prove Theorem 1. 


\subsubsection{System A}

Let

$$
\begin{aligned}
& G_{11}=\left\langle\mathcal{D} \dot{f}_{1}, \dot{f}_{1}\right\rangle, \quad G_{12}=\left\langle\mathcal{D} \dot{f}_{1}, \Delta f_{0}\right\rangle, \quad G_{13}=\left\langle\mathcal{D} \dot{f}_{1}, \Delta f_{1}\right\rangle, \\
& G_{21}=\left\langle\mathcal{D} \Delta f_{0}, \dot{f}_{1}\right\rangle, G_{22}=\left\langle\mathcal{D} \Delta f_{0}, \Delta f_{0}\right\rangle, G_{23}=\left\langle\mathcal{D} \Delta f_{0}, \Delta f_{1}\right\rangle, \\
& G_{31}=\left\langle\mathcal{D} \Delta f_{1}, \dot{f}_{1}\right\rangle, G_{32}=\left\langle\mathcal{D} \Delta f_{1}, \Delta f_{0}\right\rangle, G_{33}=\left\langle\mathcal{D} \Delta f_{1}, \Delta f_{1}\right\rangle .
\end{aligned}
$$

Denote by System $A$ the following system of differential equations

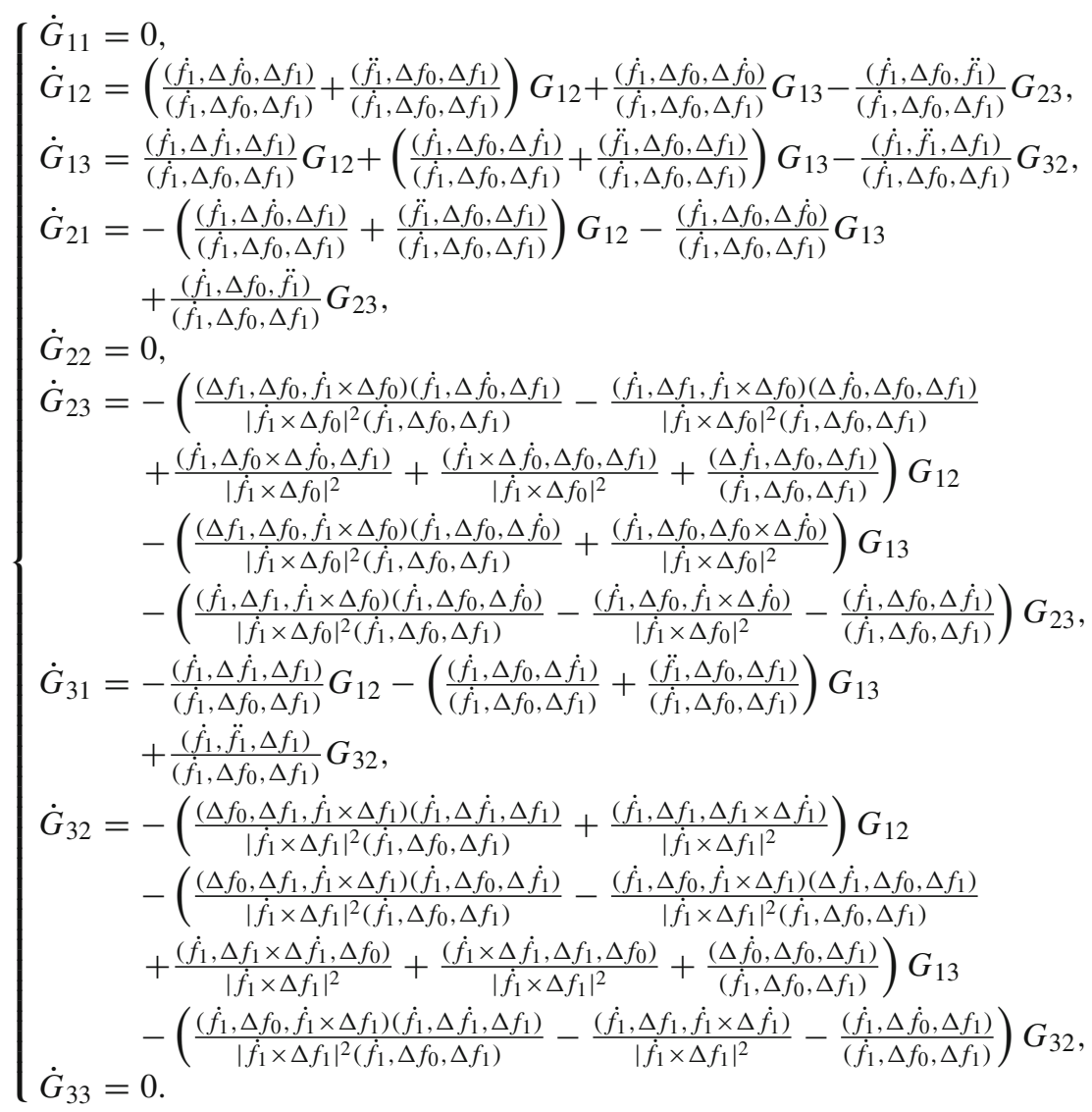

Remark 5 In Proposition 10 below we show an explicit formula for the function $G_{23}+G_{32}$, it is $\Phi$ in our notation of Sect. 3 .

Note also that $\dot{G}_{12}+\dot{G}_{21}=0$ and $\dot{G}_{13}+\dot{G}_{31}=0$ in System A.

Example 1 Let us consider a simple example of a 2-ribbon curve where $\dot{f}, \Delta f_{0}$, and $\Delta f_{1}$ are all constants. Let us call these surfaces book-shaped surfaces. Direct calculations show that

$$
\dot{G}_{11}=\dot{G}_{12}=\ldots=\dot{G}_{33}=0
$$


(this happens, since all the summands in the coefficients of System A contain either $\ddot{f_{1}}$, or $\Delta \dot{f}_{0}$, or $\Delta \dot{f}_{1}$ which are all zeroes in our case). Hence all the scalar products of the deformation with vectors $\dot{f}_{1}, \Delta f_{0}, \Delta f_{1}$ do not depend on $t$. Therefore, every element of every isometric deformations of a book-shaped surface is a book-shaped surface. Here is a typical example of isometric deformation in this class:

$$
f_{1}^{\lambda}(t)=(t, 0,0), \quad \Delta f_{0}^{\lambda}(t)=(0,1,0), \quad \Delta_{1}^{\lambda}(t)=(0, \sin \lambda, \cos \lambda) .
$$

This deformation can be geometrically seen as an opening a museum book with two rigid plastic pages.

In the following proposition we prove that for every single 2-ribbon surface $f$ (not for a deformation) and initial data for $G_{i j}$ at one point $f\left(t_{0}\right)$ System A has a unique solution. Recall that $t$ is an argument of $f$.

Proposition 3 Let $f$ be a weakly regular 2-ribbon surface in $C^{1,2,1}\left([a, b], \mathbb{R}^{3}\right)$. For every collection of initial data $G_{i j}(a)=c_{i j}$ there exists a unique solution of System $A$ on $[a, b]$.

Proof System A is the system of homogeneous linear differential equations with smooth variable coefficients (since $\left(\dot{f}_{1}, \Delta f_{0}, \Delta f_{1}\right)$ never vanishes on $\left.[a, b]\right)$ and hence for every collection of initial data it has a unique solution on the segment $[a, b]$.

\subsubsection{Every Infinitesimal Flexion Satisfies System A}

Let us show the following statement.

Proposition 4 Let $f$ be a weakly regular 2 -ribbon surface in $C^{1,2,1}\left([a, b], \mathbb{R}^{3}\right)$. Then for every infinitesimal flexion $\mathcal{D} f$ the functions $G_{11}, G_{12}, \ldots, G_{33}$ computed from the pair of semidiscrete surfaces $(f, \mathcal{D} f)$ satisfy system $A$.

We start the proof with the following general lemma.

Lemma 1 For every infinitesimal flexion $\mathcal{D} f$ we have the equalities

$$
G_{11}=G_{22}=G_{33}=0, \quad G_{12}+G_{21}=0, \text { and } G_{13}+G_{31}=0 .
$$

Proof The functions $\left|\dot{f}_{1}\right|,\left|\Delta f_{0}\right|$, and $\left|\Delta f_{1}\right|$ are infinitesimally preserved by infinitesimal flexions, hence $G_{11}, G_{22}$, and $G_{33}$ vanish.

The invariance of angles between $\dot{f}_{1}$ and $\Delta f_{0}$, and $\dot{f}_{1}$ and $\Delta f_{1}$ yield the equations $G_{12}+G_{21}=0$ and $G_{13}+G_{31}=0$, respectively.

Proof of Proposition 4. From Lemma 1 the functions $G_{11}, G_{22}$, and $G_{33}$ are zero functions, thus $\dot{G}_{11}, \dot{G}_{22}$, and $\dot{G}_{33}$ are zero functions as well.

Let us prove the expression for $\dot{G}_{12}$ and $\dot{G}_{13}$. Note that

$$
\dot{G}_{12}=\left\langle\mathcal{D} \ddot{f}_{1}, \Delta f_{0}\right\rangle+\left\langle\mathcal{D} \dot{f}_{1}, \Delta \dot{f}_{0}\right\rangle
$$


Thus Eqs. (6) and (8) imply

$$
\dot{G}_{12}=\left\langle\mathcal{D} \dot{f}_{1}, \Delta \dot{f}_{0}\right\rangle-\left\langle\ddot{f}_{1}, \mathcal{D} \Delta f_{0}\right\rangle .
$$

To obtain the expression for $\dot{G}_{12}$ rewrite $\Delta \dot{f}_{0}$ and $\ddot{f}_{1}$ in the basis consisting of vectors $\dot{f}_{1}, \Delta f_{0}$, and $\Delta f_{1}$.

$$
\begin{aligned}
\dot{G}_{12}= & \left\langle\mathcal{D} \dot{f}_{1}, \Delta \dot{f}_{0}\right\rangle-\left\langle\ddot{f}_{1}, \mathcal{D} \Delta f_{0}\right\rangle \\
= & \left(\frac{\left(\Delta \dot{f}_{0}, \Delta f_{0}, \Delta f_{1}\right)}{\left(\dot{f}_{1}, \Delta f_{0}, \Delta f_{1}\right)} G_{11}+\frac{\left(\dot{f}_{1}, \Delta \dot{f}_{0}, \Delta f_{1}\right)}{\left(\dot{f}_{1}, \Delta f_{0}, \Delta f_{1}\right)} G_{12}+\frac{\left(\dot{f}_{1}, \Delta f_{0}, \Delta \dot{f}_{0}\right)}{\left(\dot{f}_{1}, \Delta f_{0}, \Delta f_{1}\right)} G_{13}\right) \\
& -\left(\frac{\left(\ddot{f}_{1}, \Delta f_{0}, \Delta f_{1}\right)}{\left(\dot{f}_{1}, \Delta f_{0}, \Delta f_{1}\right)} G_{21}+\frac{\left(\dot{f}_{1}, \ddot{f}_{1}, \Delta f_{1}\right)}{\left(\dot{f}_{1}, \Delta f_{0}, \Delta f_{1}\right)} G_{22}+\frac{\left(\dot{f}_{1}, \Delta f_{0}, \ddot{f}_{1}\right)}{\left(\dot{f}_{1}, \Delta f_{0}, \Delta f_{1}\right)} G_{23}\right) \\
= & \left(\frac{\left(\dot{f}_{1}, \Delta \dot{f}_{0}, \Delta f_{1}\right)}{\left(\dot{f}_{1}, \Delta f_{0}, \Delta f_{1}\right)}+\frac{\left(\ddot{f}_{1}, \Delta f_{0}, \Delta f_{1}\right)}{\left(\dot{f}_{1}, \Delta f_{0}, \Delta f_{1}\right)}\right) G_{12} \\
& +\frac{\left(\dot{f}_{1}, \Delta f_{0}, \Delta \dot{f}_{0}\right)}{\left(\dot{f}_{1}, \Delta f_{0}, \Delta f_{1}\right)} G_{13}-\frac{\left(\dot{f}_{1}, \Delta f_{0}, \ddot{f}_{1}\right)}{\left(\dot{f}_{1}, \Delta f_{0}, \Delta f_{1}\right)} G_{23} .
\end{aligned}
$$

The last equation holds since $G_{11}=0, G_{22}=0$, and $G_{21}=-G_{12}$.

The same strategy works for the function $\dot{G}_{13}$.

Now we study the expressions for $\dot{G}_{21}$ and $\dot{G}_{31}$. From Lemma 1 we know that $G_{21}=-G_{12}$ and $G_{31}=-G_{13}$ and hence $\dot{G}_{21}=-\dot{G}_{12}$ and $\dot{G}_{31}=-\dot{G}_{13}$. Therefore, the equations for $\dot{G}_{21}$ and $\dot{G}_{31}$ are satisfied.

In order to get the expression for $\dot{G}_{23}$, we first show that the function $\left(\dot{f}_{1}, \Delta f_{0}, \Delta \dot{f}_{0}\right)$ is an invariant of infinitesimal flexions. Indeed,

$$
\left(\dot{f}_{1}, \Delta f_{0}, \Delta \dot{f}_{0}\right)=\left(\dot{f}_{1}, \Delta f_{0}, \dot{f}_{1}-\dot{f}_{0}\right)=-\left(\dot{f}_{1}, \Delta f_{0}, \dot{f}_{0}\right) .
$$

The vectors $\dot{f}_{0}, \dot{f}_{1}$, and $\Delta f_{0}$ form a rigid frame, hence their triple product is an invariant of infinitesimal flexions. Hence the function $\left(\dot{f}_{1}, \Delta f_{0}, \Delta \dot{f}_{0}\right)$ is an invariant as well.

The infinitesimal flexion invariance of $\left(\dot{f}_{1}, \Delta f_{0}, \Delta \dot{f}_{0}\right)$ implies that

$$
\mathcal{D}\left(\dot{f}_{1}, \Delta f_{0}, \Delta \dot{f}_{0}\right)=0 \text {. }
$$

So we get

$$
\left(\mathcal{D} \dot{f}_{1}, \Delta f_{0}, \Delta \dot{f}_{0}\right)+\left(\dot{f}_{1}, \mathcal{D} \Delta f_{0}, \Delta \dot{f}_{0}\right)+\left(\dot{f}_{1}, \Delta f_{0}, \mathcal{D} \Delta \dot{f}_{0}\right)=0 .
$$

Rewrite

$$
\begin{aligned}
\left(\dot{f}_{1}, \Delta f_{0}, \mathcal{D} \Delta \dot{f}_{0}\right)= & -\left(\mathcal{D} \dot{f}_{1}, \Delta f_{0}, \Delta \dot{f}_{0}\right)-\left(\dot{f}_{1}, \mathcal{D} \Delta f_{0}, \Delta \dot{f}_{0}\right) \\
= & -\left\langle\mathcal{D} \dot{f}_{1}, \Delta f_{0} \times \Delta \dot{f}_{0}\right\rangle+\left\langle\mathcal{D} \Delta f_{0}, \dot{f}_{1} \times \Delta \dot{f}_{0}\right\rangle \\
& -\frac{\left(\Delta f_{0} \times \Delta \dot{f}_{0}, \Delta f_{0}, \Delta f_{1}\right)}{\left(\dot{f}_{1}, \Delta f_{0}, \Delta f_{1}\right)} G_{11}-\frac{\left(\dot{f}_{1}, \Delta f_{0} \times \Delta \dot{f}_{0}, \Delta f_{1}\right)}{\left(\dot{f}_{1}, \Delta f_{0}, \Delta f_{1}\right)} G_{12} \\
= & -\frac{\left(\dot{f}_{1}, \Delta f_{0}, \Delta f_{0} \times \Delta \dot{f}_{0}\right)}{\left(\dot{f}_{1}, \Delta f_{0}, \Delta f_{1}\right)} G_{13}+\frac{\left(\dot{f}_{1} \times \Delta \dot{f}_{0}, \Delta f_{0}, \Delta f_{1}\right)}{\left(\dot{f}_{1}, \Delta f_{0}, \Delta f_{1}\right)} G_{21} \\
& +\frac{\left(\dot{f}_{1}, \dot{f}_{1} \times \Delta \dot{f}_{0}, \Delta f_{1}\right)}{\left(\dot{f}_{1}, \Delta f_{0}, \Delta f_{1}\right)} G_{22}+\frac{\left(\dot{f}_{1}, \Delta f_{0}, \dot{f}_{1} \times \Delta \dot{f}_{0}\right)}{\left(\dot{f}_{1}, \Delta f_{0}, \Delta f_{1}\right)} G_{23} .
\end{aligned}
$$


Secondly, we have

$$
\begin{aligned}
\left\langle\mathcal{D} \Delta \dot{f}_{0}, \Delta f_{0}\right\rangle & =-\left\langle\mathcal{D} \Delta f_{0}, \Delta \dot{f}_{0}\right\rangle \\
& =-\frac{\left(\Delta \dot{f}_{0}, \Delta f_{0}, \Delta f_{1}\right)}{\left(\dot{f}_{1}, \Delta f_{0}, \Delta f_{1}\right)} G_{21}-\frac{\left(\dot{f}_{1}, \Delta \dot{f}_{0}, \Delta f_{1}\right)}{\left(\dot{f}_{1}, \Delta f_{0}, \Delta f_{1}\right)} G_{22}-\frac{\left(\dot{f}_{1}, \Delta f_{0}, \Delta \dot{f}_{0}\right)}{\left(\dot{f}_{1}, \Delta f_{0}, \Delta f_{1}\right)} G_{23} .
\end{aligned}
$$

Thirdly, we get

$$
\left\langle\mathcal{D} \Delta \dot{f}_{0}, \dot{f}_{1}\right\rangle=-\left\langle\mathcal{D} \dot{f}_{1}, \Delta \dot{f}_{0}\right\rangle=-\frac{\left(\dot{f}_{1}, \Delta \dot{f}_{0}, \Delta f_{1}\right)}{\left(\dot{f}_{1}, \Delta f_{0}, \Delta f_{1}\right)} G_{12}-\frac{\left(\dot{f}_{1}, \Delta f_{0}, \Delta \dot{f}_{0}\right)}{\left(\dot{f}_{1}, \Delta f_{0}, \Delta f_{1}\right)} G_{13}
$$

Fourthly,

$$
\begin{aligned}
\left\langle\mathcal{D} \Delta \dot{f}_{0}, \Delta f_{1}\right\rangle= & \frac{\left(\Delta f_{1}, \Delta f_{0}, \dot{f}_{1} \times \Delta f_{0}\right)}{\left(\dot{f}_{1}, \Delta f_{0}, \dot{f}_{1} \times \Delta f_{0}\right)}\left\langle\mathcal{D} \Delta \dot{f}_{0}, \dot{f}_{1}\right\rangle+\frac{\left(\dot{f}_{1}, \Delta f_{1}, \dot{f}_{1} \times \Delta f_{0}\right)}{\left(\dot{f}_{1}, \Delta f_{0}, \dot{f}_{1} \times \Delta f_{0}\right)}\left\langle\mathcal{D} \Delta \dot{f}_{0}, \Delta f_{0}\right\rangle \\
& +\frac{\left(\dot{f}_{1}, \Delta f_{0}, \Delta f_{1}\right)}{\left(\dot{f}_{1}, \Delta f_{0}, \dot{f}_{1} \times \Delta f_{0}\right)}\left(\dot{f}_{1}, \Delta f_{0}, \mathcal{D} \Delta \dot{f}_{0}\right) .
\end{aligned}
$$

After the substitution of the four above expressions and simplifications we have

$$
\begin{aligned}
\langle\mathcal{D} \Delta & \left.\dot{f}_{0}, \Delta f_{1}\right\rangle \\
= & -\left(\frac{\left(\Delta f_{1}, \Delta f_{0}, \dot{f}_{1} \times \Delta f_{0}\right)\left(\dot{f}_{1}, \Delta \dot{f}_{0}, \Delta f_{1}\right)}{\left|\dot{f}_{1} \times \Delta f_{0}\right|^{2}\left(\dot{f}_{1}, \Delta f_{0}, \Delta f_{1}\right)}-\frac{\left(\dot{f}_{1}, \Delta f_{1}, \dot{f}_{1} \times \Delta f_{0}\right)\left(\Delta \dot{f}_{0}, \Delta f_{0}, \Delta f_{1}\right)}{\left|\dot{f}_{1} \times \Delta f_{0}\right|^{2}\left(\dot{f}_{1}, \Delta f_{0}, \Delta f_{1}\right)}\right. \\
& \left.+\frac{\left(\dot{f}_{1}, \Delta f_{0} \times \Delta \dot{f}_{0}, \Delta f_{1}\right)}{\left|\dot{f}_{1} \times \Delta f_{0}\right|^{2}}+\frac{\left(\dot{f}_{1} \times \Delta \dot{f}_{0}, \Delta f_{0}, \Delta f_{1}\right)}{\left|\dot{f}_{1} \times \Delta f_{0}\right|^{2}}\right) G_{12} \\
& -\left(\frac{\left(\Delta f_{1}, \Delta f_{0}, \dot{f}_{1} \times \Delta f_{0}\right)\left(\dot{f}_{1}, \Delta f_{0}, \Delta \dot{f}_{0}\right)}{\left|\dot{f}_{1} \times \Delta f_{0}\right|^{2}\left(\dot{f}_{1}, \Delta f_{0}, \Delta f_{1}\right)}+\frac{\left(\dot{f}_{1}, \Delta f_{0}, \Delta f_{0} \times \Delta \dot{f}_{0}\right)}{\left|\dot{f}_{1} \times \Delta f_{0}\right|^{2}}\right) G_{13} \\
& -\left(\frac{\left(\dot{f}_{1}, \Delta f_{1}, \dot{f}_{1} \times \Delta f_{0}\right)\left(\dot{f}_{1}, \Delta f_{0}, \Delta \dot{f}_{0}\right)}{\left|\dot{f}_{1} \times \Delta f_{0}\right|^{2}\left(\dot{f}_{1}, \Delta f_{0}, \Delta f_{1}\right)}-\frac{\left(\dot{f}_{1}, \Delta f_{0}, \dot{f}_{1} \times \Delta \dot{f}_{0}\right)}{\left|\dot{f}_{1} \times \Delta f_{0}\right|^{2}}\right) G_{23} .
\end{aligned}
$$

Further, decomposing the vector $\Delta \dot{f}_{1}$ into basis vectors $\dot{f}_{1}, \Delta f_{0}$, and $\Delta f_{1}$ we get $\left\langle\mathcal{D} \Delta f_{0}, \Delta \dot{f}_{1}\right\rangle=\frac{\left(\Delta \dot{f}_{1}, \Delta f_{0}, \Delta f_{1}\right)}{\left(\dot{f}_{1}, \Delta f_{0}, \Delta f_{1}\right)} G_{21}+\frac{\left(\dot{f}_{1}, \Delta \dot{f}_{1}, \Delta f_{1}\right)}{\left(\dot{f}_{1}, \Delta f_{0}, \Delta f_{1}\right)} G_{22}+\frac{\left(\dot{f}_{1}, \Delta f_{0}, \Delta \dot{f}_{1}\right)}{\left(\dot{f}_{1}, \Delta f_{0}, \Delta f_{1}\right)} G_{23}$.

From the last two identities, by substituting $G_{22}=0$ and $G_{21}=-G_{12}$ (see Lemma 1), we obtain the expression for

$$
\dot{G}_{23}=\frac{\partial}{\partial t}\left\langle\mathcal{D} \Delta f_{0}, \Delta f_{1}\right\rangle=\left\langle\mathcal{D} \Delta \dot{f}_{0}, \Delta f_{1}\right\rangle+\left\langle\mathcal{D} \Delta f_{0}, \Delta \dot{f}_{1}\right\rangle
$$

The expression for $\dot{G}_{32}$ is calculated in a similar way. This concludes the proof. 


\subsubsection{Existence of Infinitesimal Flexions}

Let us prove that every solution of System A with certain initial data determines an infinitesimal flexion.

Proposition 5 Let $f$ be a weakly regular 2 -ribbon surface in $C^{1,2,1}\left([a, b], \mathbb{R}^{3}\right)$. Then

(i) For an arbitrary nonzero $\alpha$ there exists a unique tangent vector $\mathcal{D} f$ at $f$ satisfying System A and the boundary conditions

$$
\mathcal{D} \dot{f}_{1}(a)=0, \quad \mathcal{D} \Delta f_{0}(a)=0, \quad \text { and } \mathcal{D} \Delta f_{1}(a)=\alpha \dot{f}_{1}(a) \times \Delta f_{1}(a)
$$

(ii) This tangent vector is an infinitesimal flexion.

Remark 6 Here and below, for a function $f$ defined on $[a, b]$ by $\dot{f}(a)$ we mean the one-sided derivative at $a$.

Proof We start with Proposition 5(i). Consider three vectors

$$
v_{1}=0, \quad v_{2}=0, \quad \text { and } \quad v_{3}=\alpha \dot{f}_{1}(a) \times \Delta f_{1}(a) .
$$

We introduce the notation

$$
\begin{aligned}
& c_{11}=\left\langle v_{1}, \dot{f}_{1}\right\rangle, c_{12}=\left\langle v_{1}, \Delta f_{0}\right\rangle, c_{13}=\left\langle v_{1}, \Delta f_{1}\right\rangle, \\
& c_{21}=\left\langle v_{2}, \dot{f}_{1}\right\rangle, c_{22}=\left\langle v_{2}, \Delta f_{0}\right\rangle, c_{23}=\left\langle v_{2}, \Delta f_{1}\right\rangle, \\
& c_{31}=\left\langle v_{3}, \dot{f}_{1}\right\rangle, c_{32}=\left\langle v_{3}, \Delta f_{0}\right\rangle, c_{33}=\left\langle v_{3}, \Delta f_{1}\right\rangle .
\end{aligned}
$$

By Proposition 3 there exists a unique solution $\left(G_{11}, G_{12}, \ldots, G_{33}\right)$ satisfying the initial conditions $G_{i j}(a)=c_{i j}$. For every point $t \in[a, b]$ the values $\mathcal{D} \dot{f}_{1}, \mathcal{D} \Delta f_{0}$, and $\mathcal{D} \Delta f_{1}$ of the tangent vector $\mathcal{D} f$ are uniquely defined in the basis $\left(\dot{f}_{1}, \Delta f_{0}, \Delta f_{1}\right)$ by Eq. (10): here we substitute the solution of System A with the initial conditions $G_{i j}(a)=c_{i j}$ to the right hand side of Eq. (10). Hence, there exists a unique tangent vector $\mathcal{D} f$ of $f$ satisfying System $\mathrm{A}$ and the boundary conditions

$$
\mathcal{D} \dot{f}_{1}(a)=0, \quad \mathcal{D} \Delta f_{1}(a)=0, \quad \text { and } \quad \mathcal{D} \Delta f_{0}(a)=\alpha \dot{f}_{1}(a) \times \Delta f_{0}(a) .
$$

This concludes the proof of the first item of the proposition.

Proof of Proposition 5(ii) By the definition of an infinitesimal flexion it is enough to check that the following 11 functions are preserved by the infinitesimal deformation:

$$
\left|\dot{f}_{i}\right|, \quad\left|\Delta f_{i}\right|, \quad\left\langle\dot{f}_{i}, \Delta f_{i-1}\right\rangle, \quad\left\langle\dot{f}_{i}, \Delta f_{i}\right\rangle, \quad \text { and }\left\langle\dot{f}_{i}, \dot{f}_{i+1}\right\rangle
$$

(for all possible admissible $i$ ).

Invariance of $\left|\dot{f}_{1}\right|,\left|\Delta f_{0}\right|,\left|\Delta f_{1}\right|,\left\langle\dot{f}_{1}, \Delta f_{0}\right\rangle$, and $\left\langle\dot{f}_{1}, \Delta f_{1}\right\rangle$.

From System A we have

$$
\dot{G}_{11}=0, \quad \dot{G}_{22}=0, \quad \dot{G}_{33}=0, \quad \dot{G}_{21}+\dot{G}_{12}=0, \quad \dot{G}_{31}+\dot{G}_{13}=0,
$$


and hence the functions

$$
\begin{gathered}
\mathcal{D}\left(\left|\dot{f}_{1}\right|^{2}\right)=2 G_{11} ; \quad \mathcal{D}\left(\left|\Delta f_{0}\right|^{2}\right)=2 G_{22} ; \quad \mathcal{D}\left(\left|\Delta f_{1}\right|^{2}\right)=2 G_{33} \\
\mathcal{D}\left\langle\dot{f}_{1}, \Delta f_{0}\right\rangle=G_{12}+G_{21}, \quad \text { and } \quad \mathcal{D}\left\langle\dot{f}_{1}, \Delta f_{1}\right\rangle=G_{31}+G_{13}
\end{gathered}
$$

are constant functions. So it is enough to show that they vanish at some point: we show this at point $a$.

$$
\begin{aligned}
& \mathcal{D}\left\langle\dot{f}_{1}(a), \dot{f}_{1}(a)\right\rangle=2\left\langle\mathcal{D} \dot{f}_{1}(a), \dot{f}_{1}(a)\right\rangle=2\left\langle 0, \dot{f}_{1}(a)\right\rangle=0 \\
& \mathcal{D}\left\langle\Delta f_{0}(a), \Delta f_{0}(a)\right\rangle=2\left\langle\mathcal{D} \Delta f_{0}(a), \Delta f_{0}(a)\right\rangle=2\left(0, \Delta f_{0}(a)\right\rangle=0 ; \\
& \mathcal{D}\left\langle\Delta f_{1}(a), \Delta f_{1}(a)\right\rangle=2\left\langle\mathcal{D} \Delta f_{1}(a), \Delta f_{1}(a)\right\rangle=2\left\langle\alpha \dot{f}_{1}(a) \times \Delta f_{1}(a), \Delta f_{1}(a)\right\rangle=0 ; \\
& \mathcal{D}\left\langle\dot{f}_{1}(a), \Delta f_{0}(a)\right\rangle=\left\langle\mathcal{D}_{\gamma} \dot{f}_{1}(a), \Delta f_{0}(a)\right\rangle+\left\langle\dot{f}_{1}(a), \mathcal{D} \Delta f_{0}(a)\right\rangle=\left\langle 0, \Delta f_{0}(a)\right\rangle \\
& +\left\langle\dot{f}_{1}(a), 0\right\rangle=0 \text {. } \\
& \mathcal{D}\left\langle\dot{f}_{1}(a), \Delta f_{1}(a)\right\rangle=\left\langle\mathcal{D} \dot{f}_{1}(a), \Delta f_{1}(a)\right\rangle+\left\langle\dot{f}_{1}(a), \mathcal{D} \Delta f_{1}(a)\right\rangle=\left\langle 0, \Delta f_{0}(a)\right\rangle \\
& +\left\langle\dot{f}_{1}(a), \alpha \dot{f}_{1}(a) \times \Delta f_{1}(a)\right\rangle=0
\end{aligned}
$$

Invariance of $\left\langle\dot{f}_{0}, \Delta f_{0}\right\rangle$ and $\left\langle\dot{f}_{2}, \Delta f_{1}\right\rangle$. Note that

$$
\left\langle\dot{f_{0}}, \Delta f_{0}\right\rangle=-\frac{1}{2} \frac{\partial}{\partial t}\left\langle\Delta f_{0}, \Delta f_{0}\right\rangle+\left\langle\dot{f}_{1}, \Delta f_{0}\right\rangle .
$$

Hence by the above item we have

$$
\mathcal{D}\left\langle\dot{f}_{0}, \Delta f_{0}\right\rangle=-\frac{1}{2} \frac{\partial}{\partial t} \mathcal{D}\left\langle\Delta f_{0}, \Delta f_{0}\right\rangle+\mathcal{D}\left\langle\dot{f}_{1}, \Delta f_{0}\right\rangle=-\frac{1}{2} \frac{\partial}{\partial t}(0)+0=0
$$

Similar reasoning shows that $\mathcal{D}\left\langle\dot{f}_{2}, \Delta f_{1}\right\rangle=0$.

Invariance of $\left\langle\dot{f}_{0}, \dot{f}_{1}\right\rangle$ and $\left\langle\dot{f}_{1}, \dot{f}_{2}\right\rangle$. Let us prove that $\mathcal{D}\left\langle\dot{f}_{0}, \dot{f}_{1}\right\rangle=0$. First, note that

$$
\begin{aligned}
\left\langle\mathcal{D} \dot{f}_{0}, \dot{f}_{1}\right\rangle & =\left\langle\mathcal{D} \dot{f}_{1}, \dot{f}_{1}\right\rangle-\left\langle\mathcal{D} \Delta \dot{f}_{0}, \dot{f}_{1}\right\rangle=-\left\langle\mathcal{D} \Delta \dot{f}_{0}, \dot{f}_{1}\right\rangle \\
& =\left\langle\mathcal{D} \Delta f_{0}, \ddot{f}_{1}\right\rangle-\frac{\partial}{\partial t}\left\langle\mathcal{D} \Delta f_{0}, \dot{f}_{1}\right\rangle .
\end{aligned}
$$

Recall that $\frac{\partial}{\partial t}\left\langle\mathcal{D} \Delta f_{0}, \dot{f}_{1}\right\rangle=\dot{G}_{21}=-\dot{G}_{12}$. Let us substitute the expression for $\dot{G}_{12}$ of System $\mathrm{A}$ and rewrite $\ddot{f}_{1}$ in the basis of vectors $\dot{f}_{1}, \Delta f_{0}$, and $\Delta f_{1}$. One obtains

$$
\begin{aligned}
\left\langle\mathcal{D} \dot{f}_{0}, \dot{f}_{1}\right\rangle & =\left\langle\mathcal{D} \Delta f_{0}, \ddot{f}_{1}\right\rangle+\dot{G}_{12} \\
& =\frac{\left(\dot{f}_{1}, \Delta \dot{f}_{0}, \Delta f_{1}\right)}{\left(\dot{f}_{1}, \Delta f_{0}, \Delta f_{1}\right)}\left\langle\mathcal{D} \dot{f}_{1}, \Delta f_{0}\right\rangle+\frac{\left(\dot{f}_{1}, \Delta f_{0}, \Delta \dot{f}_{0}\right)}{\left(\dot{f}_{1}, \Delta f_{0}, \Delta f_{1}\right)}\left\langle\mathcal{D} \dot{f}_{1}, \Delta f_{1}\right\rangle \\
& =\left\langle\mathcal{D} \dot{f}_{1}, \Delta \dot{f}_{0}\right\rangle=-\left\langle\mathcal{D} \dot{f}_{1}, \dot{f}_{0}\right\rangle .
\end{aligned}
$$


Hence

$$
\mathcal{D}\left\langle\dot{f}_{0}, \dot{f}_{1}\right\rangle=\left\langle\mathcal{D} \dot{f}_{0}, \dot{f}_{1}\right\rangle+\left\langle\mathcal{D} \dot{f}_{1}, \dot{f}_{0}\right\rangle=-\left\langle\mathcal{D} \dot{f}_{1}, \dot{f}_{0}\right\rangle+\left\langle\mathcal{D} \dot{f}_{1}, \dot{f}_{0}\right\rangle=0
$$

Therefore, $\left\langle\dot{f}_{0}, \dot{f}_{1}\right\rangle$ is invariant under the infinitesimal deformation. The proof of the invariance of $\left\langle\dot{f}_{1}, \dot{f}_{2}\right\rangle$ is analogous.

Invariance of $\left\langle\dot{f}_{0}, \dot{f}_{0}\right\rangle$ and $\left\langle\dot{f}_{2}, \dot{f}_{2}\right\rangle$. Let us prove that $\mathcal{D}\left\langle\dot{f}_{0}, \dot{f}_{0}\right\rangle=0$.

$$
\mathcal{D}\left\langle\dot{f}_{0}, \dot{f}_{0}\right\rangle=2\left\langle\mathcal{D} \dot{f}_{0}, \dot{f}_{0}\right\rangle=2\left\langle\mathcal{D} \Delta \dot{f}_{0}, \Delta \dot{f}_{0}\right\rangle+2 \mathcal{D}\left\langle\dot{f}_{1}, \dot{f}_{0}\right\rangle-2\left\langle\mathcal{D} \dot{f}_{1}, \dot{f}_{1}\right\rangle
$$

We have already shown that $\mathcal{D}\left\langle\dot{f}_{1}, \dot{f}_{0}\right\rangle=0$ and $\left\langle\mathcal{D} \dot{f}_{1}, \dot{f}_{1}\right\rangle=0$. Hence

$$
\mathcal{D}\left\langle\dot{f}_{0}, \dot{f}_{0}\right\rangle=2\left\langle\mathcal{D} \Delta \dot{f}_{0}, \Delta \dot{f}_{0}\right\rangle
$$

We rewrite the last $\Delta \dot{f}_{0}$ in the last expression in the basis $\dot{f}_{1}, \Delta f_{0}, \dot{f}_{1} \times \Delta f_{0}$ and get

$$
\begin{aligned}
\left(\mathcal{D} \Delta \dot{f}_{0}, \Delta \dot{f_{0}}\right\rangle= & \frac{\left(\Delta \dot{f}_{0}, \Delta f_{0}, \dot{f}_{1} \times \Delta f_{0}\right)}{\left(\dot{f}_{1}, \Delta f_{0}, \dot{f}_{1} \times \Delta f_{0}\right)}\left\langle\mathcal{D} \Delta \dot{f}_{0}, \dot{f}_{1}\right\rangle+\frac{\left(\dot{f}_{1}, \Delta \dot{f}_{0}, \dot{f}_{1} \times \Delta f_{0}\right)}{\left(\dot{f}_{1}, \Delta f_{0}, \dot{f}_{1} \times \Delta f_{0}\right)}\left\langle\mathcal{D} \Delta \dot{f}_{0}, \Delta f_{0}\right\rangle \\
& +\frac{\left(\dot{f}_{1}, \Delta f_{0}, \Delta \dot{f}_{0}\right)}{\left(\dot{f}_{1}, \Delta f_{0}, \dot{f}_{1} \times \Delta f_{0}\right)}\left(\mathcal{D} \Delta \dot{f}_{0}, \dot{f}_{1}, \Delta f_{0}\right) .
\end{aligned}
$$

Let us rewrite $\left\langle\mathcal{D} \Delta \dot{f}_{0}, \dot{f}_{1}\right\rangle,\left\langle\mathcal{D} \Delta \dot{f}_{0}, \Delta f_{0}\right\rangle$, and $\left(\mathcal{D} \Delta \dot{f}_{0}, \dot{f}_{1}, \Delta f_{0}\right)$ in terms of $G_{11}, \ldots, G_{33}$. First, we have:

$$
\left\langle\mathcal{D} \Delta \dot{f}_{0}, \dot{f}_{1}\right\rangle=\left\langle\mathcal{D} \dot{f}_{0}, \dot{f}_{1}\right\rangle=-\left\langle\mathcal{D} \dot{f}_{1}, \dot{f}_{0}\right\rangle=-\left\langle\mathcal{D} \dot{f}_{1}, \Delta \dot{f}_{0}\right\rangle
$$

The second equality holds since we have shown that $\mathcal{D}\left\langle\dot{f}_{0}, \dot{f}_{1}\right\rangle=0$. If we rewrite $\Delta \dot{f}_{0}$ in the basis $\dot{f}_{1}, \Delta f_{0}, \Delta f_{1}$, we get the following:

$$
\left\langle\mathcal{D} \Delta \dot{f}_{0}, \dot{f}_{1}\right\rangle=-\left\langle\mathcal{D} \dot{f}_{1}, \Delta \dot{f}_{0}\right\rangle=-\frac{\left(\dot{f}_{1}, \Delta \dot{f}_{0}, \Delta f_{1}\right)}{\left(\dot{f}_{1}, \Delta f_{0}, \Delta f_{1}\right)} G_{12}-\frac{\left(\dot{f}_{1}, \Delta f_{0}, \Delta \dot{f}_{0}\right)}{\left(\dot{f}_{1}, \Delta f_{0}, \Delta f_{1}\right)} G_{13}
$$

Secondly, we have

$$
\left\langle\mathcal{D} \Delta \dot{f}_{0}, \Delta f_{0}\right\rangle=-\left\langle\mathcal{D} \Delta f_{0}, \Delta \dot{f}_{0}\right\rangle=\frac{\left(\Delta \dot{f}_{0}, \Delta f_{0}, \Delta f_{1}\right)}{\left(\dot{f}_{1}, \Delta f_{0}, \Delta f_{1}\right)} G_{12}-\frac{\left(\dot{f}_{1}, \Delta f_{0}, \Delta \dot{f}_{0}\right)}{\left(\dot{f}_{1}, \Delta f_{0}, \Delta f_{1}\right)} G_{23} .
$$

Thirdly, with

$$
\begin{aligned}
\dot{G}_{23}-\left\langle\mathcal{D} \Delta f_{0}, \Delta \dot{f}_{1}\right\rangle= & \left\langle\mathcal{D} \Delta \dot{f}_{0}, \Delta f_{1}\right\rangle=\frac{\left(\Delta f_{1}, \Delta f_{0}, \dot{f}_{1} \times \Delta f_{0}\right)}{\left(\dot{f}_{1}, \Delta f_{0}, \dot{f}_{1} \times \Delta f_{0}\right)}\left\langle\mathcal{D} \Delta \dot{f}_{0}, \dot{f}_{1}\right\rangle \\
& +\frac{\left(\dot{f}_{1}, \Delta f_{1}, \dot{f}_{1} \times \Delta f_{0}\right)}{\left(\dot{f}_{1}, \Delta f_{0}, \dot{f}_{1} \times \Delta f_{0}\right)}\left\langle\mathcal{D} \Delta \dot{f}_{0}, \Delta f_{0}\right\rangle \\
& +\frac{\left(\dot{f}_{1}, \Delta f_{0}, \Delta f_{1}\right)}{\left(\dot{f}_{1}, \Delta f_{0}, \dot{f}_{1} \times \Delta f_{0}\right)}\left(\mathcal{D} \Delta \dot{f}_{0}, \dot{f}_{1}, \Delta f_{0}\right) .
\end{aligned}
$$


and the expression for $\dot{G}_{23}$ of System A we get:

$$
\begin{aligned}
\left(\mathcal{D} \Delta \dot{f}_{0}, \dot{f}_{1}, \Delta f_{0}\right)= & -\left(\frac{\left(\dot{f}_{1} \times \Delta \dot{f}_{0}, \Delta f_{0}, \Delta f_{1}\right)}{\left(\dot{f}_{1}, \Delta f_{0}, \Delta f_{1}\right)}+\frac{\left(\dot{f}_{1}, \Delta f_{0} \times \Delta \dot{f}_{0}, \Delta f_{1}\right)}{\left(\dot{f}_{1}, \Delta f_{0}, \Delta f_{1}\right)}\right) G_{12} \\
& -\frac{\left(\dot{f}_{1}, \Delta f_{0}, \Delta f_{0} \times \Delta \dot{f}_{0}\right)}{\left(\dot{f}_{1}, \Delta f_{0}, \Delta f_{1}\right)} G_{13}+\frac{\left(\dot{f}_{1}, \Delta f_{0}, \dot{f}_{1} \times \Delta \dot{f}_{0}\right)}{\left(\dot{f}_{1}, \Delta f_{0}, \Delta f_{1}\right)} G_{23} .
\end{aligned}
$$

Finally, we substitute the obtained last three expressions for

$$
\left\langle\mathcal{D} \Delta \dot{f}_{0}, \dot{f}_{1}\right\rangle, \quad\left\langle\mathcal{D} \Delta \dot{f}_{0}, \Delta f_{0}\right\rangle, \quad \text { and } \quad\left(\mathcal{D} \Delta \dot{f}_{0}, \dot{f}_{1}, \Delta f_{0}\right)
$$

respectively to Expression (11) and arrive at

$\left\langle\mathcal{D} \Delta \dot{f}_{0}, \Delta \dot{f}_{0}\right\rangle$

$$
\begin{aligned}
=( & -\frac{\left(\Delta \dot{f}_{0}, \Delta f_{0}, \dot{f}_{1} \times \Delta f_{0}\right)\left(\dot{f}_{1}, \Delta \dot{f}_{0}, \Delta f_{1}\right)}{\left(\dot{f}_{1}, \Delta f_{0}, \dot{f}_{1} \times \Delta f_{0}\right)\left(\dot{f}_{1}, \Delta f_{0}, \Delta f_{1}\right)}+\frac{\left(\dot{f}_{1}, \Delta \dot{f}_{0}, \dot{f}_{1} \times \Delta f_{0}\right)\left(\Delta \dot{f}_{0}, \Delta f_{0}, \Delta f_{1}\right)}{\left(\dot{f}_{1}, \Delta f_{0}, \dot{f}_{1} \times \Delta f_{0}\right)\left(\dot{f}_{1}, \Delta f_{0}, \Delta f_{1}\right)} \\
& \left.-\frac{\left(\dot{f}_{1}, \Delta f_{0}, \Delta \dot{f}_{0}\right)\left(\dot{f}_{1} \times \Delta \dot{f}_{0}, \Delta f_{0}, \Delta f_{1}\right)}{\left(\dot{f}_{1}, \Delta f_{0}, \dot{f}_{1} \times \Delta f_{0}\right)\left(\dot{f}_{1}, \Delta f_{0}, \Delta f_{1}\right)}-\frac{\left(\dot{f}_{1}, \Delta f_{0}, \Delta \dot{f}_{0}\right)\left(\dot{f}_{1}, \Delta f_{0} \times \Delta \dot{f}_{0}, \Delta f_{1}\right)}{\left(\dot{f}_{1}, \Delta f_{0}, \dot{f}_{1} \times \Delta f_{0}\right)\left(\dot{f}_{1}, \Delta f_{0}, \Delta f_{1}\right)}\right) G_{12} \\
& +\left(-\frac{\left(\Delta \dot{f}_{0}, \Delta f_{0}, \dot{f}_{1} \times \Delta f_{0}\right)\left(\dot{f}_{1}, \Delta f_{0}, \Delta \dot{f}_{0}\right)}{\left(\dot{f}_{1}, \Delta f_{0}, \dot{f}_{1} \times \Delta f_{0}\right)\left(\dot{f}_{1}, \Delta f_{0}, \Delta f_{1}\right)}-\frac{\left(\dot{f}_{1}, \Delta f_{0}, \Delta \dot{f}_{0}\right)\left(\dot{f}_{1}, \Delta f_{0}, \Delta f_{0} \times \Delta \dot{f}_{0}\right)}{\left(\dot{f}_{1}, \Delta f_{0}, \dot{f}_{1} \times \Delta f_{0}\right)\left(\dot{f}_{1}, \Delta f_{0}, \Delta f_{1}\right)}\right) G_{13} \\
& +\left(-\frac{\left(\dot{f}_{1}, \Delta \dot{f}_{0}, \dot{f}_{1} \times \Delta f_{0}\right)\left(\dot{f}_{1}, \Delta f_{0}, \Delta \dot{f}_{0}\right)}{\left(\dot{f}_{1}, \Delta f_{0}, \dot{f}_{1} \times \Delta f_{0}\right)\left(\dot{f}_{1}, \Delta f_{0}, \Delta f_{1}\right)}+\frac{\left(\dot{f}_{1}, \Delta f_{0}, \Delta \dot{f}_{0}\right)\left(\dot{f}_{1}, \Delta f_{0}, \dot{f}_{1} \times \Delta \dot{f}_{0}\right)}{\left(\dot{f}_{1}, \Delta f_{0}, \dot{f}_{1} \times \Delta f_{0}\right)\left(\dot{f}_{1}, \Delta f_{0}, \Delta f_{1}\right)}\right) G_{23} .
\end{aligned}
$$

It is clear that the coefficients of $G_{13}$ and $G_{23}$ vanish identically. Let us study the coefficient of $G_{12}$.

Consider the following mixed product $\left(\Delta \dot{f}_{0}, \Delta \dot{f}_{0}, \dot{f}_{1} \times \Delta f_{0}\right)$, it is identical to zero. Let us rewrite $\Delta \dot{f}_{0}$ in the second position of the mixed product in the basis $\dot{f}_{0}, \Delta f_{0}$, $\Delta f_{1}$. We get the relation

$$
\begin{gathered}
\frac{\left(\Delta \dot{f}_{0}, \Delta f_{0}, \Delta f_{1}\right)}{\left(\dot{f}_{1}, \Delta f_{0}, \Delta f_{1}\right)}\left(\Delta \dot{f}_{0}, \dot{f}_{1}, \dot{f}_{1} \times \Delta f_{0}\right)+\frac{\left(\dot{f}_{1}, \Delta \dot{f}_{0}, \Delta f_{1}\right)}{\left(\dot{f}_{1}, \Delta f_{0}, \Delta f_{1}\right)}\left(\Delta \dot{f}_{0}, \Delta f_{0}, \dot{f}_{1} \times \Delta f_{0}\right) \\
=-\frac{\left(\dot{f}_{1}, \Delta f_{0}, \Delta \dot{f}_{0}\right)}{\left(\dot{f}_{1}, \Delta f_{0}, \Delta f_{1}\right)}\left(\Delta \dot{f}_{0}, \Delta f_{1}, \dot{f}_{1} \times \Delta f_{0}\right)
\end{gathered}
$$

We apply this identity to the first two summands of the coefficient of $G_{12}$ and get the following expression for the coefficient of $G_{12}$ :

$$
\begin{aligned}
& \frac{\left(\dot{f}_{1}, \Delta f_{0}, \Delta \dot{f}_{0}\right)\left(\Delta \dot{f}_{0}, \Delta f_{1}, \dot{f}_{1} \times \Delta f_{0}\right)}{\left(\dot{f}_{1}, \Delta f_{0}, \Delta f_{1}\right)\left|\dot{f}_{1} \times \Delta f_{0}\right|^{2}}-\frac{\left(\dot{f}_{1}, \Delta f_{0} \times \Delta \dot{f}_{0}, \Delta f_{1}\right)\left(\dot{f}_{1}, \Delta f_{0}, \Delta \dot{f}_{0}\right)}{\left(\dot{f}_{1}, \Delta f_{0}, \Delta f_{1}\right)\left|\dot{f}_{1} \times \Delta f_{0}\right|^{2}} \\
& -\frac{\left(\dot{f}_{1} \times \Delta \dot{f}_{0}, \Delta f_{0}, \Delta f_{1}\right)\left(\dot{f}_{1}, \Delta f_{0}, \Delta \dot{f}_{0}\right)}{\left(\dot{f}_{1}, \Delta f_{0}, \Delta f_{1}\right)\left|\dot{f}_{1} \times \Delta f_{0}\right|^{2}}
\end{aligned}
$$


We rewrite this as

$$
\begin{gathered}
\frac{\left(\dot{f}_{1}, \Delta f_{0}, \Delta \dot{f}_{0}\right)}{\left(\dot{f}_{1}, \Delta f_{0}, \Delta f_{1}\right)\left|\dot{f}_{1} \times \Delta f_{0}\right|^{2}}\left(\left(\Delta \dot{f}_{0}, \Delta f_{1}, \dot{f}_{1} \times \Delta f_{0}\right)-\left(\dot{f}_{1}, \Delta f_{0} \times \Delta \dot{f}_{0}, \Delta f_{1}\right)\right. \\
\left.-\left(\dot{f}_{1} \times \Delta \dot{f}_{0}, \Delta f_{0}, \Delta f_{1}\right)\right) .
\end{gathered}
$$

Let us study the expression in the brackets.

$$
\begin{aligned}
& \left(\Delta \dot{f}_{0}, \Delta f_{1}, \dot{f}_{1} \times \Delta f_{0}\right)-\left(\dot{f}_{1}, \Delta f_{0} \times \Delta \dot{f}_{0}, \Delta f_{1}\right)-\left(\dot{f}_{1} \times \Delta \dot{f}_{0}, \Delta f_{0}, \Delta f_{1}\right) \\
& \quad=-\left(\Delta \dot{f}_{0} \times\left(\dot{f}_{1} \times \Delta f_{0}\right)+\dot{f}_{1} \times\left(\Delta f_{0} \times \Delta \dot{f}_{0}\right)+\Delta f_{0} \times\left(\Delta \dot{f}_{0} \times \dot{f}_{1}\right), \Delta f_{1}\right)=\left(0, \Delta f_{1}\right)=0 .
\end{aligned}
$$

The second equality holds by the Jacobi identity. Hence the coefficient of $G_{12}$ is zero. Therefore,

$$
\mathcal{D}\left\langle\dot{f}_{0}, \dot{f}_{0}\right\rangle=2\left\langle\mathcal{D} \Delta \dot{f}_{0}, \Delta \dot{f}_{0}\right\rangle=0
$$

and $\left\langle\dot{f}_{0}, \dot{f}_{0}\right\rangle$ is invariant under the infinitesimal deformation.

The proof of the invariance of $\left\langle\dot{f}_{2}, \dot{f}_{2}\right\rangle$ repeats the proof for $\left\langle\dot{f}_{0}, \dot{f}_{0}\right\rangle$.

So we have checked the invariance of all the 11 functions in the definition of an infinitesimal flexion. Hence $\mathcal{D} f$ is an infinitesimal flexion.

Now we have all the ingredients to prove the main theorem of this subsection.

\subsubsection{Conclusion of the Proof of Theorem 1}

Existence The existence of an infinitesimal flexion follows directly from Proposition 5(i).

Uniqueness By Proposition 4 every infinitesimal flexion satisfies System A. Since we consider 2-ribbon surfaces with fixed initial position, for every non-zero infinitesimal flexion $\mathcal{D} f$ we have:

$$
\mathcal{D} \dot{f}_{1}(a)=0, \quad \mathcal{D} \Delta f_{0}(a)=0, \quad \text { and } \quad \mathcal{D} \Delta f_{1}(a)=\alpha \dot{f}_{1}(a) \times \Delta f_{1}(a)
$$

for some non-zero $\alpha$. Hence by Proposition 5 this is one of the flexions of Proposition 5(i). So the set of infinitesimal flexions is one-dimensional. Since the set is a linear space, it is a line. Hence $f$ has one degree of infinitesimal flexibility.

Theorem 1 together with Proposition 5 imply the following.

Corollary 1 Let $f \in C_{0}^{1,2,1}\left([a, b], \mathbb{R}^{3}\right)$ be a weakly regular 2-ribbon surface with fixed initial position, and let $\mathcal{D} f$ be its infinitesimal flexion satisfying

$$
\mathcal{D} \dot{f}_{1}(a)=0, \quad \mathcal{D} \Delta f_{1}(a)=0, \quad \text { and } \mathcal{D} \Delta f_{0}(a)=0
$$

Then $\mathcal{D} f=0$. 


\subsection{Operators Related to Infinitesimal Flexions}

Let us fix an orthonormal basis $\left(e_{1}, e_{2}, e_{3}\right)$ in $\mathbb{R}^{3}$. Denote by $\Omega_{3 \times 3}^{1}$ the Banach space

$$
\left(\left(C^{1}[a, b]\right)^{3}\right)^{3} \cong\left(C^{1}[a, b]\right)^{9}
$$

with the norm

$$
\left\|\left(h_{11}, h_{12}, \ldots, h_{33}\right)\right\|=\max _{1 \leq i, j \leq 3}\left(\max \left(\sup \left|h_{i j}\right|, \sup \left|\dot{h}_{i j}\right|\right)\right) .
$$

Consider the following map

$$
Z: C^{1,2,1}\left([a, b], \mathbb{R}^{3}\right) \rightarrow \Omega_{3 \times 3}^{1},
$$

where for a 2-ribbon surface $f$ the image $Z(f)$ in the basis $\left(e_{1}, e_{2}, e_{3}\right)$ is defined as

$$
\begin{aligned}
\dot{f}_{1}(t) & =\left(h_{11}(t), h_{12}(t), h_{13}(t)\right), \\
\Delta f_{0}(t) & =\left(h_{21}(t), h_{22}(t), h_{23}(t)\right), \\
\Delta f_{1}(t) & =\left(h_{31}(t), h_{32}(t), h_{33}(t)\right) .
\end{aligned}
$$

Note that every 2-ribbon surface $f$ is defined by $\dot{f}_{1}, \Delta f_{0}$, and $\Delta f_{1}$ up to a translation. So after fixing, say, $f_{1}(a)=(0,0,0)$ one has a bijection.

We say that a point $h=\left(h_{11}, h_{12}, \ldots, h_{33}\right)$ in $\Omega_{3 \times 3}^{1}$ is in general position if the determinant

$$
\operatorname{det}\left(\begin{array}{lll}
h_{11} & h_{12} & h_{13} \\
h_{21} & h_{22} & h_{23} \\
h_{31} & h_{32} & h_{33}
\end{array}\right) \neq 0
$$

for every $t \in[a, b]$. This condition obviously corresponds to the weak regularity condition, i.e., to

$$
\left(\dot{f}_{1}, \Delta f_{0}, \Delta f_{1}\right) \neq 0
$$

Denote by $\Sigma_{\Omega}$ the set of all points $h$ that are not in general position.

Definition 17 Denote by $\mathcal{V}^{ \pm}:[0, \Lambda] \times\left(\Omega_{3 \times 3}^{1} \backslash \Sigma_{\Omega}\right) \rightarrow \Omega_{3 \times 3}^{1}$ two operators of infinitesimal flexion in coordinates $\left(h_{11}, h_{12}, \ldots, h_{33}\right)$ :

$$
\begin{aligned}
\mathcal{V}_{l-1, m}^{ \pm}(\lambda, h)= & \frac{\left(e_{m}, \Delta f_{0}, \Delta f_{1}\right)}{\left(\dot{f}_{1}, \Delta f_{0}, \Delta f_{1}\right)} G_{l-1,1}(h)+\frac{\left(\dot{f}_{1}, e_{m}, \Delta f_{1}\right)}{\left(\dot{f}_{1}, \Delta f_{0}, \Delta f_{1}\right)} G_{l-1,2}(h) \\
& +\frac{\left(\dot{f}_{1}, \Delta f_{0}, e_{m}\right)}{\left(\dot{f}_{1}, \Delta f_{0}, \Delta f_{1}\right)} G_{l-1,3}(h) .
\end{aligned}
$$

for $(1 \leq l, m \leq 3)$. Here $G_{11}(h), G_{12}(h), \ldots, G_{33}(h)$ is a solution of System A at point $f$ with the initial conditions corresponding to

$$
\mathcal{D} \dot{f}_{1}(a)=0, \quad \mathcal{D} \Delta f_{0}(a)=0, \quad \text { and } \mathcal{D} \Delta f_{1}(a)= \pm \dot{f}_{1}(a) \times \Delta f_{1}(a),
$$


i.e.,

$$
\begin{array}{ll}
G_{11}(a)=0, G_{12}(a)=0, & G_{13}(a)=0 \\
G_{21}(a)=0, G_{22}(a)=0, & G_{23}(a)=0 \\
G_{31}(a)=0, G_{32}(a)= \pm\left(\dot{f}_{1}(a), \Delta f_{0}(a), \Delta f_{1}(a)\right), G_{33}(a)=0
\end{array}
$$

(Here we take "+" sign for $\mathcal{V}^{+}$and “-” for $\mathcal{V}^{-}$.)

Note that both $\mathcal{V}^{+}$and $\mathcal{V}^{-}$are autonomous operators, they do not depend on time parameter $\lambda$.

It is important that the following statement holds.

Proposition 6 Let $h$ be a point of $\Omega_{3 \times 3}^{1}$ in general position and $\lambda \in[0, \Lambda]$. Then we have

$$
\mathcal{V}^{ \pm}(\lambda, h) \in \Omega_{3 \times 3}^{1}
$$

Proof The proof is straightforward, all functions involved in Expression (12) are continuously differentiable, and hence both $\mathcal{V}^{+}(\lambda, h)$ and $\mathcal{V}^{-}(\lambda, h)$ are continuously differentiable.

Remark 7 Let us show in brief how to find the coordinates of the infinitesimal deformation $\mathcal{D} f$ in the basis $e_{1}, e_{2}, e_{3}$ satisfying

$$
\begin{gathered}
\mathcal{D} f_{1}(a)=0, \quad \mathcal{D} \dot{f}_{1}(a)=0, \quad \mathcal{D} \Delta f_{0}(a)=0, \\
\text { and } \mathcal{D} \Delta f_{1}(a)=\dot{f}_{1}(a) \times \Delta f_{1}(a)
\end{gathered}
$$

First, one should solve System A with the above initial data, then substitute the obtained solution $\left(G_{11}, G_{12}, \ldots, G_{33}\right)$ to Eq. (12). Now we have the coordinates of $\mathcal{D} \dot{f}_{1}, \mathcal{D} \Delta f_{0}$, and $\mathcal{D} \Delta f_{1}$. Having the additional condition $\mathcal{D} f_{1}(a)=0$ one can construct $\mathcal{D} f_{1}, \mathcal{D} f_{0}$, and $\mathcal{D} f_{2}$ :

$$
\mathcal{D} f_{1}\left(t_{0}\right)=\int_{a}^{t_{0}} \mathcal{D} \dot{f}_{1}(t) d(t), \quad \mathcal{D} f_{0}=\mathcal{D} f_{1}-\mathcal{D} \Delta f_{0}, \quad \mathcal{D} f_{2}=\mathcal{D} f_{1}+\mathcal{D} \Delta f_{1} .
$$

Further we will work in the following subspace of $\Omega_{3 \times 3}^{1}$. Denote

$$
\tilde{\Omega}_{3 \times 3}^{1}=\left\{h \in \Omega_{3 \times 3}^{1} \mid h_{12}(a)=h_{13}(a)=h_{23}(a)=0\right\} .
$$

It is clear that $\tilde{\Omega}_{3 \times 3}^{1}$ is a Banach space itself.

We have the following important property of $\tilde{\Omega}_{3 \times 3}^{1}$.

Proposition 7 For every $\lambda \in[0, \Lambda]$ and $h \in \tilde{\Omega}_{3 \times 3}^{1} \backslash \Sigma_{\Omega}$ the subspace $\tilde{\Omega}_{3 \times 3}^{1}$ is an invariant space of the operators $\mathcal{V}^{+}(\lambda, h)$ and $\mathcal{V}^{-}(\lambda, h)$. 
Proof From the conditions

$$
\mathcal{D} \dot{f}_{1}(a)=0, \quad \text { and } \mathcal{D} \Delta f_{0}(a)=0
$$

we have $G_{i j}(a)=0$ for all $i=1,2$, and $j=1,2,3$. Hence by Expression (12)

$$
\mathcal{V}_{11}^{ \pm}(\lambda, h)(a)=\mathcal{V}_{12}^{ \pm}(\lambda, h)(a)=\ldots=\mathcal{V}_{23}^{ \pm}(\lambda, h)(a)=0
$$

for all $\lambda \in[0, \Lambda]$ and $h \in \Omega_{3 \times 3}^{1}$. Therefore, for every $\lambda \in[0, \Lambda]$ and $h \in \tilde{\Omega}_{3 \times 3}^{1} \backslash \Sigma_{\Omega}$ we have $\mathcal{V}^{ \pm}(\lambda, h) \in \tilde{\Omega}_{3 \times 3}^{1}$.

Finally we have the following important statement.

Proposition 8 The map $Z$ is a bijection of $\tilde{\Omega}_{3 \times 3}^{1}$ and $C_{0}^{1,2,1}\left([a, b], \mathbb{R}^{3}\right)$.

Proof The inverse map $Z^{-1}(h)=\left(f_{0}, f_{1}, f_{2}\right)$ is defined as

$$
\begin{aligned}
& f_{1}\left(t_{0}\right)=\int_{a}^{t_{0}}\left(\begin{array}{l}
h_{11}(t) \\
h_{12}(t) \\
h_{13}(t)
\end{array}\right) d t, \quad f_{0}\left(t_{0}\right)=f_{1}\left(t_{0}\right)-\left(\begin{array}{l}
h_{21}\left(t_{0}\right) \\
h_{22}\left(t_{0}\right) \\
h_{23}\left(t_{0}\right)
\end{array}\right), \\
& f_{2}\left(t_{0}\right)=\left(\begin{array}{l}
h_{31}\left(t_{0}\right) \\
h_{32}\left(t_{0}\right) \\
h_{33}\left(t_{0}\right)
\end{array}\right)-f_{1}\left(t_{0}\right) .
\end{aligned}
$$

at every $t_{0} \in[a, b]$.

\subsection{Finite Flexibility of 2-Ribbon Surfaces}

In Sect. 3.2 we showed that every 2-ribbon surface in general position is infinitesimally flexible and that the space of its infinitesimal flexions is one-dimensional. The aim of this subsection is to show that a weakly regular 2-ribbon surface is finitely flexible and has one degree of finite flexibility.

\subsubsection{Lipschitz Condition}

We start with the discussion of the initial value problem for the following two differential equations on the set of all points $\tilde{\Omega}_{3 \times 3}^{1}$ in general position (here $\lambda$ is the time parameter):

$$
\frac{\partial h}{\partial \lambda}=\mathcal{V}^{+}(\lambda, h) \text { and } \frac{\partial h}{\partial \lambda}=\mathcal{V}^{-}(\lambda, h)
$$

To solve the initial value problem we study local Lipschitz properties for $\mathcal{V}^{+}$and $\mathcal{V}^{-}$.

Definition 18 Consider a Banach space $E$ with a norm $|*|_{E}$, and a positive real number $\Lambda$. Let $U$ be a subset of $[0, \Lambda] \times E$. We say that a functional $\mathcal{F}: U \rightarrow E$ locally 
satisfies a Lipschitz condition if for every point $\left(\lambda_{0}, p\right)$ in $U$ there exist a neighborhood $V$ of the point and a constant $K$ such that for every pair of points $\left(\lambda, p_{1}\right)$ and $\left(\lambda, p_{2}\right)$ in $V$ the inequality

$$
\left|\mathcal{F}\left(\lambda, p_{1}\right)-\mathcal{F}\left(\lambda, p_{2}\right)\right|_{E} \leq K\left|p_{1}-p_{2}\right|_{E}
$$

holds.

First we verify a Lipschitz condition for the following operator. Define $\mathcal{G}:[0, \Lambda] \times$ $\tilde{\Omega}_{3 \times 3}^{1} \rightarrow \tilde{\Omega}_{3 \times 3}^{1}$ by

$$
\mathcal{G}_{i j}(\lambda, h)=G_{i j}(h), \quad 1 \leq i, j \leq 3
$$

where $G_{i j}(h)$ are defined by Eq. (10).

Lemma 2 For every point $h \in U$ in general position, there exists a neighborhood $V_{h}$ of $h$ such that the functional $\mathcal{G}$ locally satisfies a Lipschitz condition in $[0, \Lambda] \times V_{h}$.

Proof Consider a point $h \in U$. The element $\left(G_{11}, G_{12}, \ldots, G_{33}\right)$ itself satisfies a system of linear differential equations (System A). The coefficients of this system depend only on a point of $\tilde{\Omega}_{3 \times 3}^{1}$. Since the point $h$ is in general position, there exists a positive real constant $K$ such that for a sufficiently small neighborhood $V_{h}$ of $h$ the dependence is $K$-Lipschitz, i.e., for $p$ and $q$ from $V_{h}$ every coefficient $c$ of System A satisfies the inequality

$$
|c(p)-c(q)|<K\|p-q\|
$$

Hence the solutions for $t \in[a, b]$ satisfy the Lipschitz condition for a fixed initial data on $V_{h}$. (This is clear from the fact that the solution of the system with small coefficients $c(p)-c(q)$ will be almost constant, the difference in each coordinate will not be greater than $9(b-a) K\|p-q\|$.) Finally the solution for $t \in[a, b]$ satisfies the Lipschitz condition for a fixed parameter and different initial data on $V_{h}$ (See Proposition 1.10.1 in Cartan 1967). Therefore, for some constants $\bar{K}_{l}$ we have

$$
\sup \left(\left|G_{i j}(p)-G_{i j}(q)\right|\right)<\bar{K}_{i j}\|p-q\|, \quad 1 \leq i, j \leq 3 .
$$

From System A we know that the $\dot{G}_{i, j}$ linearly depend on $G_{11}, G_{12}, \ldots, G_{33}$, therefore, we get the Lipschitz condition for the derivatives: for some constants $\tilde{K}_{l}$ we have

$$
\sup \left(\left|\dot{G}_{i j}(p)-\dot{G}_{i j}(q)\right|\right)<\tilde{K}_{i j}\|p-q\|, \quad 1 \leq i, j \leq 3
$$

Thus there exists a real number $\hat{K}$ such that for all points $p$ and $q$ in $V_{h}$,

$$
\begin{aligned}
\|\mathcal{G}(\lambda, p)-\mathcal{G}(\lambda, q)\|= & \max _{1 \leq i, j \leq 3}\left(\operatorname { m a x } \left(\sup \left|G_{i j}(p)-G_{i j}(q)\right|,\right.\right. \\
& \left.\left.\sup \left|\dot{G}_{i j}(p)-\dot{G}_{i j}(q)\right|\right)\right)<\hat{K}\|p-q\| .
\end{aligned}
$$


Thus $\mathcal{G}$ satisfies a Lipschitz condition on $V_{h}$. Therefore, $\mathcal{G}$ satisfies a Lipschitz condition on $[0, \Lambda] \times V_{h}$ (since $\mathcal{G}$ is autonomous).

Lemma 2 and Expression (12) directly imply the following statement.

Corollary 2 For every point $h \in U$ in general position and, there exists a neighborhood $V_{h}$ of $h$ such that both functionals $\mathcal{V}^{+}$and $\mathcal{V}^{-}$locally satisfy a Lipschitz. condition in $[0, \Lambda] \times V_{h}$.

\subsubsection{Existence and Uniqueness of Solutions}

Let us prove the following general statement.

Proposition 9 Let $h_{0} \in \tilde{\Omega}_{3 \times 3}^{1}$ be in general position. Then for sufficiently small positive $\varepsilon$ there exists a unique solution $\gamma$ of the equation

$$
\frac{\partial h}{\partial \lambda}=\mathcal{V}^{+}(\lambda, h)
$$

on $[-\varepsilon, \varepsilon]$, such that $\gamma(0)=h_{0}$.

We start with the following general lemma.

Lemma 3 Let $h_{0} \in \tilde{\Omega}_{3 \times 3}^{1}$ be in general position. A deformation $\gamma$ with $\gamma(0)=h_{0}$ is a solutions of Eq. (15) if and only if $\gamma$ satisfies

$$
\frac{\partial \gamma}{\partial \lambda}= \begin{cases}\mathcal{V}^{+}(\lambda, \gamma(\lambda)) & \text { for all } \lambda \in[0, \Lambda], \\ -\mathcal{V}^{-}(-\lambda, \gamma(\lambda)) & \text { for all } \lambda \in[-\Lambda, 0]\end{cases}
$$

Proof The proof of the Lemma is straightforward.

Proof of Proposition 9 As we showed in Corollary 2, the operators $\mathcal{V}^{+}$and $\mathcal{V}^{-}$satisfy a Lipschitz condition in some neighborhood of the point $Z^{-1}(f)$. From the general theory of differential equations on Banach spaces (see for instance the first section of the second chapter of Cartan 1967) it follows that this condition implies local existence and uniqueness of a solution of the initial value problem for the differential Eq. (14). Hence by Lemma 3 for a sufficiently small positive $\varepsilon$ there exists a unique solution $\gamma$ of Eq. (15) on $[-\varepsilon, \varepsilon]$ satisfying the condition $\gamma(0)=h_{0}$.

\subsubsection{Finite Flexibility}

The key point for finite flexibility of 2-ribbon surfaces is the following lemma.

Lemma 4 Let $\left\{f^{\lambda}\right\}, \lambda \in[-\varepsilon, \varepsilon]$ for some $\varepsilon>0$, be a normalized isometric deformation in the space $C_{0}^{1,2,1}\left([a, b], \mathbb{R}^{3}\right)$ such that all 2-ribbon surfaces of the family are weakly regular. Let also $\left\{Z^{-1}\left(f^{\lambda}\right)\right\}$ be the corresponding deformation in $\tilde{\Omega}_{3 \times 3}^{1}$. Then $\left\{f^{\lambda}\right\}$ is an isometric deformation if and only $\left\{Z^{-1}\left(f^{\lambda}\right)\right\}$ satisfies Eq. (15) for all $\lambda \in[-\varepsilon, \varepsilon]$. 
Proof Let $\gamma=\left\{f^{\lambda}\right\}, \lambda \in[-\varepsilon, \varepsilon]$, be a normalized isometric deformation in $C_{0}^{1,2,1}\left([a, b], \mathbb{R}^{3}\right)$ such that all 2-ribbon surfaces of the family are weakly regular. Every normalized deformation satisfies Condition (13) at every point $\lambda \in[-\varepsilon, \varepsilon]$ with the positive choice of the sign.

Since $\gamma$ is an isometric deformation, $\mathcal{D}_{\gamma} f^{\lambda}$ is an infinitesimal flexion at every point $\lambda \in[-\varepsilon, \varepsilon]$. Hence the corresponding functions $G_{i j}^{\lambda}$ satisfy system A (by Proposition 4). Let us now write $\mathcal{D}_{\gamma} \dot{f}_{1}^{\lambda}, \mathcal{D}_{\gamma} \Delta f_{0}^{\lambda}$, and $\mathcal{D}_{\gamma} \Delta f_{1}^{\lambda}$ in the basis $e_{1}, e_{2}, e_{3}$ using functions $G_{i j}^{\lambda}$. Recall that

$$
\begin{aligned}
\mathcal{D}_{\gamma} \dot{f}_{1}^{\lambda} & =\left(\mathcal{D}_{\gamma} \dot{f}_{1}^{\lambda}, \dot{f}_{1}^{\lambda}\right) \dot{f}_{1}^{\lambda}+\left(\mathcal{D}_{\gamma} \dot{f}_{1}^{\lambda}, \Delta f_{0}^{\lambda}\right) \Delta f_{0}^{\lambda}+\left(\mathcal{D}_{\gamma} \dot{f}_{1}^{\lambda}, \Delta f_{1}^{\lambda}\right) \Delta f_{1}^{\lambda} \\
& =G_{11} \dot{f}_{1}^{\lambda}+G_{12} \Delta f_{0}^{\lambda}+G_{13} \Delta f_{1}^{\lambda} .
\end{aligned}
$$

Hence in the basis $\left(e_{1}, e_{2}, e_{3}\right)$ we have

$$
\begin{aligned}
\frac{\partial \dot{f}_{1}^{\lambda}}{\partial \lambda} & =\mathcal{D}_{\gamma} \dot{f}_{1}^{\lambda} \\
& =\sum_{m=1}^{3}\left(\frac{\left(e_{m}, \Delta f_{0}^{\lambda}, \Delta f_{1}^{\lambda}\right)}{\left(\dot{f}_{1}^{\lambda}, \Delta f_{0}^{\lambda}, \Delta f_{1}^{\lambda}\right)} G_{11}^{\lambda}+\frac{\left(\dot{f}_{1}^{\lambda}, e_{m}, \Delta f_{1}^{\lambda}\right)}{\left(\dot{f}_{1}^{\lambda}, \Delta f_{0}^{\lambda}, \Delta f_{1}^{\lambda}\right)} G_{12}^{\lambda}+\frac{\left(\dot{f}_{1}^{\lambda}, \Delta f_{0}^{\lambda}, e_{m}\right)}{\left(\dot{f}_{1}^{\lambda}, \Delta f_{0}^{\lambda}, \Delta f_{1}^{\lambda}\right)} G_{13}^{\lambda}\right) e_{m} \\
& =\sum_{m=1}^{3}\left(\mathcal{V}_{1, m}^{+}\left(\lambda, Z^{-1}\left(f^{\lambda}\right)\right)\right) e_{m} .
\end{aligned}
$$

Similarly we have:

$$
\begin{aligned}
& \frac{\partial \Delta f_{0}^{\lambda}}{\partial \lambda}=\sum_{m=1}^{3}\left(\mathcal{V}_{2, m}^{+}\left(\lambda, Z^{-1}\left(f^{\lambda}\right)\right)\right) e_{m}, \\
& \frac{\partial \Delta f_{1}^{\lambda}}{\partial \lambda}=\sum_{m=1}^{3}\left(\mathcal{V}_{3, m}^{+}\left(\lambda, Z^{-1}\left(f^{\lambda}\right)\right)\right) e_{m} .
\end{aligned}
$$

Hence by Definition 17 the corresponding derivatives in the space $\tilde{\Omega}_{3 \times 3}^{1}$ satisfy:

$$
\frac{\partial Z^{-1}\left(f^{\lambda}\right)}{\partial \lambda}=\mathcal{V}^{+}\left(\lambda, Z^{-1}\left(f^{\lambda}\right)\right)
$$

for every $\lambda \in[-\varepsilon, \varepsilon]$.

Conversely, let $Z^{-1}\left(f^{\lambda}\right)$ satisfy

$$
\frac{\partial Z^{-1}\left(f^{\lambda}\right)}{\partial \lambda}=\mathcal{V}^{+}\left(\lambda, Z^{-1}\left(f^{\lambda}\right)\right)
$$

for every $\lambda \in[-\varepsilon, \varepsilon]$. Then the corresponding $\mathcal{D}_{\gamma} \dot{f}_{1}^{\lambda}, \mathcal{D}_{\gamma} \Delta f_{0}^{\lambda}$, and $\mathcal{D}_{\gamma} \Delta f_{1}^{\lambda}$ are defined as in (16) and (17). Hence the correspondent scalar products $G_{i j}^{\lambda}$ satisfy System A for 
$\lambda \in[-\varepsilon, \varepsilon]$. Thus $\mathcal{D}_{\gamma} f^{\lambda}$ is an infinitesimal flexion for every $\lambda \in[-\varepsilon, \varepsilon]$. Hence by $\left\{f^{\lambda}\right\}$ is an isometric deformation on $[-\varepsilon, \varepsilon]$. From construction it follows that $\left\{f^{\lambda}\right\}$ is a normalized deformation.

Now we prove the following theorem on finite flexibility of weakly regular 2-ribbon surfaces.

Theorem 2 Every 2-ribbon weakly regular semidiscrete surface $f$ in the space $C_{0}^{1,2,1}\left([a, b], \mathbb{R}^{3}\right)$ has one degree of finite flexibility.

Proof On the one hand, by Lemma 4 normalized isometric deformations of $f$ with a fixed initial position are in one-to-one correspondence with Solution of Eq. (15) satisfying $\gamma(0)=Z^{-1}(f)$. On the other hand, by Proposition 9 for sufficiently small positive $\varepsilon$ there exists a unique solution $\gamma$ of Eq. (15) satisfying $\gamma(0)=Z^{-1}(f)$. Hence, there exists a unique normalized isometric deformations of $f$ (with the parameter in $[-\varepsilon, \varepsilon]$ for sufficiently small positive $\varepsilon$ ). Therefore, $f$ has one degree of finite flexibility.

Remark 8 In fact, one can prove the statement of Theorem 2 for the spaces of functions $C_{0}^{m, m+1, m}\left([a, b], \mathbb{R}^{3}\right)$ for arbitrary $m \geq 1$. We are not going to use this later so we omit the details here. The proofs mostly repeat the ones for the case $m=1$ shown in details above.

\section{Infinitesimal Flexibility of 3-Ribbon Surfaces}

In this section we find necessary infinitesimal flexibility condition of 3-ribbon surfaces. For the case of $n$-ribbon surfaces each 3-ribbon subsurface gives a condition of infinitesimal flexibility.

\subsection{Preliminary Statements on Infinitesimal Flexion of 3-Ribbon Surfaces}

In this subsection we prove certain relations that we use further in the proof of the statement on infinitesimal flexibility condition for 3-ribbon surfaces.

Consider the following function

$$
\Phi=\left\langle\Delta f_{0}, \Delta f_{1}\right\rangle
$$

This function plays a central role in our further description of the infinitesimal flexibility condition of 3-ribbon and $n$-ribbon surfaces (see Theorems 3 and 7). Let $\mathcal{D} \Phi$ be the infinitesimal flexion of $\Phi$. Via the function $\mathcal{D} \Phi$ we describe monodromy conditions for finite flexibility. Proposition 10 and Corollary 3 deliver necessary tools to describe continuous and discrete parts of the monodromy condition on $\Phi$.

Remark 9 In the proofs of the statements of this subsection we fix the flexion of the initial frame $\left(\dot{f}_{1}(a), \Delta f_{0}(a), \Delta f_{1}(a)\right)$ in the following way

$$
\mathcal{D} \dot{f}_{1}=\mathcal{D} \Delta f_{1}\left(t_{0}\right)=0
$$


(compare to the space $C_{0}^{M}[a, b], \mathbb{R}^{3}$ where $\mathcal{D} \dot{f}_{1}(a)=\mathcal{D} \Delta f_{0}(a)=0$ instead), as usual we fix the point $f_{1}(a)$ as well. This simplifies calculations for the 3-ribbon surfaces, since the fixed bar with endpoints $f_{1}(a)$ and $f_{2}(a)$ belongs to the middle strip.

\subsubsection{Continuous Shift}

Here we study the dependence of the infinitesimal flexion $\mathcal{D} \Phi$ on the argument $t$.

Proposition 10 (On continuous shift.) Let $f$ be a weakly regular 2-ribbon surface in $C^{1,2,1}\left([a, b], \mathbb{R}^{3}\right)$. Then for every infinitesimal flexion $\mathcal{D} \Phi$ the following condition holds:

$$
\mathcal{D} \Phi\left(t_{2}\right)=\mathcal{D} \Phi\left(t_{1}\right) \cdot \exp \left(\int_{t_{1}}^{t_{2}} \frac{\left(\dot{f}_{1}, \Delta \dot{f}_{0}, \Delta f_{1}\right)+\left(\dot{f}_{1}, \Delta f_{0}, \Delta \dot{f}_{1}\right)}{\left(\dot{f}_{1}, \Delta f_{0}, \Delta f_{1}\right)} d t\right) .
$$

This is a direct consequence of the next lemma.

Lemma 5 Let $f$ be a weakly regular 2-ribbon surface in $C^{1,2,1}\left([a, b], \mathbb{R}^{3}\right)$, then

$$
\mathcal{D} \dot{\Phi}=\frac{\left(\dot{f}_{1}, \Delta \dot{f}_{0}, \Delta f_{1}\right)+\left(\dot{f}_{1}, \Delta f_{0}, \Delta \dot{f}_{1}\right)}{\left(\dot{f}_{1}, \Delta f_{0}, \Delta f_{1}\right)} \mathcal{D} \Phi .
$$

Proof Note that

$$
\begin{aligned}
& \mathcal{D} \Phi=\left\langle\mathcal{D} \Delta f_{0}, \Delta f_{1}\right\rangle+\left\langle\Delta f_{0}, \mathcal{D} \Delta f_{1}\right\rangle, \quad \text { and } \\
& \mathcal{D} \dot{\Phi}=\left\langle\mathcal{D} \Delta \dot{f}_{0}, \Delta f_{1}\right\rangle+\left\langle\mathcal{D} \Delta f_{0}, \Delta \dot{f}_{1}\right\rangle+\left\langle\Delta \dot{f}_{0}, \mathcal{D} \Delta f_{1}\right\rangle+\left\langle\Delta f_{0}, \mathcal{D} \Delta \dot{f}_{1}\right\rangle .
\end{aligned}
$$

Let us prove the statement of the lemma for an arbitrary point $t_{0}$. Without loss of generality we fix $\mathcal{D} \dot{f}_{1}\left(t_{0}\right)=0$ and $\mathcal{D} \Delta f_{1}\left(t_{0}\right)=0$ (this is possible since every flexion is isometric to a flexion with such properties and isometries of flexions do not change the functions in the formula of the lemma). Then $\mathcal{D} \Delta f_{0}\left(t_{0}\right)$ is proportional to $\dot{f}_{1}\left(t_{0}\right) \times \Delta f_{0}\left(t_{0}\right)$, and hence there exists some real number $\alpha$ with

$$
\mathcal{D} \Delta f_{0}\left(t_{0}\right)=\alpha \dot{f}_{1}\left(t_{0}\right) \times \Delta f_{0}\left(t_{0}\right)
$$

Thus we immediately get

$$
\mathcal{D} \Phi\left(t_{0}\right)=\left\langle\mathcal{D} \Delta f_{0}(t), \Delta f_{1}(t)\right\rangle=\alpha\left(\dot{f}_{1}\left(t_{0}\right), \Delta f_{0}\left(t_{0}\right), \Delta f_{1}\left(t_{0}\right)\right) .
$$

Let us express the summands for $\mathcal{D} \dot{\Phi}\left(t_{0}\right)$. We start with $\left\langle\mathcal{D} \Delta \dot{f}_{0}\left(t_{0}\right), \Delta f_{1}\left(t_{0}\right)\right\rangle$. First we note that

$$
\begin{aligned}
\Delta f_{1}= & \frac{\left(\Delta f_{1}, \Delta f_{0}, f_{1} \times \Delta \dot{f}_{0}\right)}{\left(\dot{f}_{1}, \Delta f_{0}, \dot{f}_{1} \times \Delta f_{0}\right)} \dot{f}_{1}+\frac{\left(\dot{f}_{1}, \Delta f_{1}, f_{1} \times \Delta \dot{f}_{0}\right)}{\left(\dot{f}_{1}, \Delta f_{0}, \dot{f}_{1} \times \Delta f_{0}\right)} \Delta f_{0} \\
& +\frac{\left(\dot{f}_{1}, \Delta f_{0}, \Delta f_{1}\right)}{\left(\dot{f}_{1}, \Delta f_{0}, \dot{f}_{1} \times \Delta f_{0}\right)} f_{1} \times \Delta \dot{f}_{0} .
\end{aligned}
$$


Equation (6) implies

$$
\left\langle\mathcal{D} \Delta \dot{f}_{0}\left(t_{0}\right), \dot{f}_{1}\left(t_{0}\right)\right\rangle=-\left\langle\mathcal{D} \dot{f}_{1}\left(t_{0}\right), \Delta \dot{f}_{0}\left(t_{0}\right)\right\rangle=-\left\langle 0, \Delta \dot{f}_{0}\left(t_{0}\right)\right\rangle=0
$$

From Eq. (4) we have

$$
\begin{aligned}
\left\langle\mathcal{D} \Delta \dot{f}_{0}\left(t_{0}\right), \Delta f_{0}\left(t_{0}\right)\right\rangle & =-\left\langle\mathcal{D} \Delta f_{0}\left(t_{0}\right), \Delta \dot{f}_{0}\left(t_{0}\right)\right\rangle \\
& =-\alpha\left(\dot{f}_{1}\left(t_{0}\right), \Delta f_{0}\left(t_{0}\right), \Delta \dot{f}_{0}\left(t_{0}\right)\right)
\end{aligned}
$$

The function $\left(\Delta \dot{f}_{0}, \dot{f}_{1}, \Delta f_{0}\right)$ is invariant of an infinitesimal flexion, therefore:

$$
\left(\mathcal{D} \Delta \dot{f}_{0}, \dot{f}_{1}, \Delta f_{0}\right)+\left(\Delta \dot{f}_{0}, \mathcal{D} \dot{f}_{1}, \Delta f_{0}\right)+\left(\Delta \dot{f}_{0}, \dot{f}_{1}, \mathcal{D} \Delta f_{0}\right)=0
$$

and hence

$$
\begin{aligned}
\left\langle\mathcal{D} \Delta \dot{f}_{0}\left(t_{0}\right), \dot{f}_{1}\left(t_{0}\right) \times \Delta f_{0}\left(t_{0}\right)\right\rangle & =-\left(\Delta \dot{f}_{0}\left(t_{0}\right), \dot{f}_{1}\left(t_{0}\right), \mathcal{D} \Delta f_{0}\left(t_{0}\right)\right) \\
& =-\alpha\left(\Delta \dot{f}_{0}\left(t_{0}\right), \dot{f}_{1}\left(t_{0}\right), \dot{f}_{1}\left(t_{0}\right) \times \Delta f_{0}\left(t_{0}\right)\right)
\end{aligned}
$$

Now we decompose $\Delta \dot{f}_{0}\left(t_{0}\right)$ in the last formula in the basis of vectors $\dot{f}_{1}\left(t_{0}\right), \Delta f_{0}\left(t_{0}\right)$, and $\Delta f_{1}\left(t_{0}\right)$ :

$$
\begin{aligned}
(\Delta & \left.\dot{f}_{0}\left(t_{0}\right), \dot{f}_{1}\left(t_{0}\right), \dot{f}_{1}\left(t_{0}\right) \times \Delta f_{0}\left(t_{0}\right)\right) \\
= & \frac{\left(\dot{f}_{1}\left(t_{0}\right), \Delta \dot{f}_{0}\left(t_{0}\right), \Delta f_{1}\left(t_{0}\right)\right)}{\left(\dot{f}_{1}\left(t_{0}\right), \Delta f_{0}\left(t_{0}\right), \Delta f_{1}\left(t_{0}\right)\right)}\left(\Delta f_{0}\left(t_{0}\right), \dot{f}_{1}\left(t_{0}\right), \dot{f}_{1}\left(t_{0}\right) \times \Delta f_{0}\left(t_{0}\right)\right) \\
& +\frac{\left(\dot{f}_{1}\left(t_{0}\right), \Delta f_{0}\left(t_{0}\right), \Delta \dot{f}_{0}\left(t_{0}\right)\right)}{\left(\dot{f}_{1}\left(t_{0}\right), \Delta f_{0}\left(t_{0}\right), \Delta f_{1}\left(t_{0}\right)\right)}\left(\Delta f_{1}\left(t_{0}\right), \dot{f}_{1}\left(t_{0}\right), \dot{f}_{1}\left(t_{0}\right) \times \Delta f_{0}\left(t_{0}\right)\right) .
\end{aligned}
$$

Therefore, after substitution (18) of $\Delta f_{2}$ we apply (18b), (18c), (18d), and the last expression and get

$$
\begin{aligned}
\langle\mathcal{D} \Delta & \left.\dot{f}_{0}\left(t_{0}\right), \Delta f_{1}\left(t_{0}\right)\right\rangle \\
= & -\alpha \frac{\left(\dot{f}_{1}\left(t_{0}\right), \Delta f_{1}\left(t_{0}\right), \dot{f}_{1}\left(t_{0}\right) \times \Delta f_{0}\left(t_{0}\right)\right)}{\left(\dot{f}_{1}\left(t_{0}\right), \Delta f_{0}\left(t_{0}\right), \dot{f}_{1}\left(t_{0}\right) \times \Delta f_{0}\left(t_{0}\right)\right)}\left(\dot{f}_{1}\left(t_{0}\right), \Delta f_{0}\left(t_{0}\right), \Delta \dot{f}_{0}\left(t_{0}\right)\right) \\
& -\alpha \frac{\left(\dot{f}_{1}\left(t_{0}\right), \Delta \dot{f}_{0}\left(t_{0}\right), \Delta f_{1}\left(t_{0}\right)\right)}{\left(\dot{f}_{1}\left(t_{0}\right), \Delta f_{0}\left(t_{0}\right), \dot{f}_{1}\left(t_{0}\right) \times \Delta f_{0}\left(t_{0}\right)\right)}\left(\Delta f_{0}\left(t_{0}\right), \dot{f}_{1}\left(t_{0}\right), \dot{f}_{1}\left(t_{0}\right) \times \Delta f_{0}\left(t_{0}\right)\right) \\
& -\alpha \frac{\left(\dot{f}_{1}\left(t_{0}\right), \Delta f_{0}\left(t_{0}\right), \Delta \dot{f}_{0}\left(t_{0}\right)\right)}{\left(\dot{f}_{1}\left(t_{0}\right), \Delta f_{0}\left(t_{0}\right), \dot{f}_{1}\left(t_{0}\right) \times \Delta f_{0}\left(t_{0}\right)\right)}\left(\Delta f_{1}\left(t_{0}\right), \dot{f}_{1}\left(t_{0}\right), \dot{f}_{1}\left(t_{0}\right) \times \Delta f_{0}\left(t_{0}\right)\right) \\
= & -\alpha\left(\dot{f}_{1}\left(t_{0}\right), \Delta f_{1}\left(t_{0}\right), \Delta \dot{f}_{0}\left(t_{0}\right)\right) .
\end{aligned}
$$


Similar calculations for the summand $\left\langle\Delta f_{0}\left(t_{0}\right), \mathcal{D} \Delta \dot{f}_{1}\left(t_{0}\right)\right\rangle$ (applying Eqs. (3), (5), and (7) and the conditions $\mathcal{D} \dot{f}_{1}\left(t_{0}\right)=0$ and $\mathcal{D} \Delta f_{1}\left(t_{0}\right)=0$ ) show that

$$
\left\langle\Delta f_{0}\left(t_{0}\right), \mathcal{D} \Delta \dot{f}_{1}\left(t_{0}\right)\right\rangle=0
$$

Further we have

$$
\begin{aligned}
& \left\langle\mathcal{D} \Delta f_{0}\left(t_{0}\right), \Delta \dot{f}_{1}\left(t_{0}\right)\right\rangle=\alpha\left(\dot{f}_{1}\left(t_{0}\right), \Delta f_{0}\left(t_{0}\right), \Delta \dot{f}_{1}\left(t_{0}\right)\right), \\
& \left\langle\Delta \dot{f}_{0}\left(t_{0}\right), \mathcal{D} \Delta f_{1}\left(t_{0}\right)\right\rangle=0 .
\end{aligned}
$$

Therefore,

$$
\mathcal{D} \dot{\Phi}\left(t_{0}\right)=\alpha\left(\left(\dot{f}_{1}\left(t_{0}\right), \Delta \dot{f}_{0}\left(t_{0}\right), \Delta f_{1}\left(t_{0}\right)\right)+\left(\dot{f}_{1}\left(t_{0}\right), \Delta f_{0}\left(t_{0}\right), \Delta \dot{f}_{1}\left(t_{0}\right)\right)\right),
$$

and consequently

$$
\mathcal{D} \dot{\Phi}\left(t_{0}\right)=\frac{\left(\dot{f}_{1}\left(t_{0}\right), \Delta \dot{f}_{0}\left(t_{0}\right), \Delta f_{1}\left(t_{0}\right)\right)+\left(\dot{f}_{1}\left(t_{0}\right), \Delta f_{0}\left(t_{0}\right), \Delta \dot{f}_{1}\left(t_{0}\right)\right)}{\left(\dot{f}_{1}\left(t_{0}\right), \Delta f_{0}\left(t_{0}\right), \Delta f_{1}\left(t_{0}\right)\right)} \mathcal{D} \Phi\left(t_{0}\right) .
$$

Thus Lemma 5 holds for all possible values of $t_{0}$.

\subsubsection{Discrete Shift}

Every 3-ribbon surface contains two 2-ribbon surfaces as a subsurfaces. Each of them has an infinitesimal flexion $\mathcal{D} \Phi_{i}(i=1,2)$, where

$$
\Phi_{1}=\left\langle\Delta f_{0}, \Delta f_{1}\right\rangle \quad \text { and } \quad \Phi_{2}=\left\langle\Delta f_{1}, \Delta f_{2}\right\rangle
$$

Let us show the relation between $\mathcal{D} \Phi_{1}$ and $\mathcal{D} \Phi_{2}$ for the same values of argument $t$.

First, in Proposition 11 we show a relation for $\mathcal{D}\left\langle\ddot{f}_{1}, \ddot{f}_{1}\right\rangle$ and $\mathcal{D}\left\langle\ddot{f}_{2}, \ddot{f}_{2}\right\rangle$. Secondly, in Proposition 12 we give a link between $\mathcal{D}\left\langle\ddot{f}_{1}, \ddot{f}_{1}\right\rangle$ and $\mathcal{D} \Phi_{1}$. This will result in the formula of Corollary 3 on the relation between $\mathcal{D} \Phi_{1}$ and $\mathcal{D} \Phi_{2}$.

We start with a formula expressing $\mathcal{D}\left\langle\ddot{f}_{2}, \ddot{f}_{2}\right\rangle$ via $\mathcal{D}\left\langle\ddot{f}_{1}, \ddot{f}_{1}\right\rangle$.

Proposition 11 Let $f$ be a strongly regular 3-ribbon surface in the space $C^{1,2,2,1}$ $\left([a, b], \mathbb{R}^{3}\right)$, and let $\mathcal{D} f$ be its infinitesimal flexion. Then the following equation holds:

$$
\mathcal{D}\left\langle\ddot{f}_{2}, \ddot{f}_{2}\right\rangle=\frac{\left(\dot{f}_{2}, \ddot{f}_{2}, \Delta f_{1}\right)}{\left(\dot{f}_{1}, \ddot{f}_{1}, \Delta f_{1}\right)} \mathcal{D}\left\langle\ddot{f}_{1}, \ddot{f}_{1}\right\rangle \text {. }
$$

Proof We do calculations at a point $t_{0}$ again assuming that $\mathcal{D} \dot{f}_{1}\left(t_{0}\right)=0$ and $\mathcal{D} \Delta f_{1}\left(t_{0}\right)=0$ (by choosing an appropriate isometric representative of the deformation). Let us show that $\mathcal{D} \dot{f}_{2}\left(t_{0}\right)=0$. First, note that

$$
\mathcal{D} \dot{f}_{2}\left(t_{0}\right)=\mathcal{D} \dot{f}_{1}\left(t_{0}\right)+\mathcal{D} \Delta \dot{f}_{1}\left(t_{0}\right)=\mathcal{D} \Delta \dot{f}_{1}\left(t_{0}\right)
$$


Secondly we show that the inner products of $\mathcal{D} \Delta \dot{f}_{1}\left(t_{0}\right)$ and the vectors $\dot{f}_{1}\left(t_{0}\right), \Delta f_{1}\left(t_{0}\right)$, and $\dot{f}_{1}\left(t_{0}\right) \times \Delta f_{1}\left(t_{0}\right)$ are all zero (this would imply that $\mathcal{D} \Delta \dot{f}_{1}\left(t_{0}\right)=0$ ).

From Eq. (7) we have

$$
\left\langle\mathcal{D} \Delta \dot{f}_{1}\left(t_{0}\right), \dot{f}_{1}\left(t_{0}\right)\right\rangle=-\left\langle\mathcal{D} \dot{f}_{1}\left(t_{0}\right), \Delta \dot{f}_{1}\left(t_{0}\right)\right\rangle=-\left\langle 0, \Delta \dot{f}_{1}\left(t_{0}\right)\right\rangle=0
$$

Further, from Eq. (5), we get

$$
\left\langle\mathcal{D} \Delta \dot{f}_{1}\left(t_{0}\right), \Delta f_{1}\left(t_{0}\right)\right\rangle=-\left\langle\mathcal{D} \Delta f_{1}\left(t_{0}\right), \Delta \dot{f}_{1}\left(t_{0}\right)\right\rangle=0 .
$$

Finally, from the equation $\mathcal{D}\left(\dot{f}_{1}, \Delta f_{1}, \Delta \dot{f}_{1}\right)=0$ we obtain

$$
\begin{aligned}
& \left\langle\mathcal{D} \Delta \dot{f}_{1}\left(t_{0}\right), \dot{f}_{1}\left(t_{0}\right) \times \Delta f_{1}\left(t_{0}\right)\right\rangle \\
& \quad=-\left(\Delta \dot{f}_{1}\left(t_{0}\right), \mathcal{D} \dot{f}_{1}\left(t_{0}\right), \Delta f_{1}\left(t_{0}\right)\right)-\left(\Delta \dot{f}_{1}\left(t_{0}\right), \dot{f}_{1}\left(t_{0}\right), \mathcal{D} \Delta f_{1}\left(t_{0}\right)\right)=0 .
\end{aligned}
$$

Therefore, $\mathcal{D} \Delta \dot{f}_{1}\left(t_{0}\right)=0$, and hence $\mathcal{D} \dot{f}_{2}\left(t_{0}\right)=0$.

From Eqs. (1) and (9) we get

$$
\begin{aligned}
\left\langle\mathcal{D} \ddot{f}_{1}\left(t_{0}\right), \dot{f}_{1}\left(t_{0}\right)\right\rangle & =\frac{\partial}{\partial t}\left\langle\mathcal{D} \dot{f}_{1}\left(t_{0}\right), \dot{f}_{1}\left(t_{0}\right)\right\rangle-\left\langle\ddot{f}_{1}\left(t_{0}\right), \mathcal{D} \dot{f}_{1}\left(t_{0}\right)\right\rangle \\
& =0-\left\langle\ddot{f}_{1}\left(t_{0}\right), 0\right\rangle=0 ; \\
\left\langle\mathcal{D} \ddot{f}_{1}\left(t_{0}\right), \Delta f_{1}\left(t_{0}\right)\right\rangle & =-\left\langle\ddot{f}_{1}\left(t_{0}\right), \mathcal{D} \Delta f_{1}\left(t_{0}\right)\right\rangle=-\left\langle\ddot{f}_{1}\left(t_{0}\right), 0\right\rangle=0 .
\end{aligned}
$$

Therefore, for some real number $\beta_{1}$ we have

$$
\mathcal{D} \ddot{f}_{1}\left(t_{0}\right)=\beta_{1} \dot{f}_{1}\left(t_{0}\right) \times \Delta f_{1}\left(t_{0}\right) \text {. }
$$

By a similar reasoning (since we have shown that $\mathcal{D} \dot{f}_{2}\left(t_{0}\right)=0$ ) we get

$$
\mathcal{D} \ddot{f}_{2}\left(t_{0}\right)=\beta_{2} \dot{f}_{2}\left(t_{0}\right) \times \Delta f_{1}\left(t_{0}\right) .
$$

Since $\frac{\partial}{\partial t}\left(\mathcal{D}\left(\dot{f}_{1}, \Delta f_{1}, \dot{f}_{2}\right)\right)=0$, at point $t_{0}$ we have

$$
\left(\mathcal{D} \ddot{f}_{1}\left(t_{0}\right), \Delta f_{1}\left(t_{0}\right), \dot{f}_{2}\left(t_{0}\right)\right)+\left(\dot{f}_{1}\left(t_{0}\right), \Delta f_{1}\left(t_{0}\right), \mathcal{D} \ddot{f}_{2}\left(t_{0}\right)\right)=0 .
$$

Hence,

$$
\beta_{1}\left(\dot{f}_{1}\left(t_{0}\right) \times \Delta f_{1}\left(t_{0}\right), \Delta f_{1}\left(t_{0}\right), \dot{f}_{2}\left(t_{0}\right)\right)+\beta_{2}\left(\dot{f}_{1}\left(t_{0}\right), \Delta f_{1}\left(t_{0}\right), \dot{f}_{2}\left(t_{0}\right) \times \Delta f_{1}\left(t_{0}\right)\right)=0,
$$

and, therefore $\beta_{1}=\beta_{2}$. This implies

$$
\mathcal{D}\left\langle\ddot{f}_{1}\left(t_{0}\right), \ddot{f}_{1}\left(t_{0}\right)\right\rangle=2\left\langle\mathcal{D} \ddot{f}_{1}\left(t_{0}\right), \ddot{f}\left(t_{0}\right)\right\rangle=2 \beta_{1}\left(\dot{f}_{1}\left(t_{0}\right), \Delta f_{1}\left(t_{0}\right), \ddot{f}_{1}\left(t_{0}\right)\right)
$$

and

$$
\mathcal{D}\left\langle\ddot{f}_{2}\left(t_{0}\right), \ddot{f}_{2}\left(t_{0}\right)\right\rangle=2 \beta_{1}\left(\dot{f}_{2}\left(t_{0}\right), \Delta f_{1}\left(t_{0}\right), \ddot{f}_{2}\left(t_{0}\right)\right)
$$


The last two formulas imply the statement of Proposition 11.

Now let us relate $\mathcal{D}\left\langle\ddot{f}_{1}, \ddot{f}_{1}\right\rangle$ and $\mathcal{D} \Phi$.

Proposition 12 Let us consider a weakly regular 2-ribbon surface $f$ in the space $C^{1,2,1}\left([a, b], \mathbb{R}^{3}\right)$. Then the following identity holds:

$$
\mathcal{D}\left\langle\ddot{f}_{1}, \ddot{f}_{1}\right\rangle=2 \frac{\left(\dot{f}_{1}, \ddot{f}_{1}, \Delta f_{0}\right)\left(\dot{f}_{1}, \ddot{f}_{1}, \Delta f_{1}\right)}{\left(\dot{f}_{1}, \Delta f_{0}, \Delta f_{1}\right)^{2}} \mathcal{D} \Phi .
$$

Proof We restrict ourselves to the case of a point. Without loss of generality we assume that $\mathcal{D} \dot{f}_{1}\left(t_{0}\right)=0$ and $\mathcal{D} \Delta f_{1}\left(t_{0}\right)=0$. So as we have seen before, there exists $\alpha$ such that

$$
\mathcal{D} \Delta f_{0}\left(t_{0}\right)=\alpha \dot{f}_{1}\left(t_{0}\right) \times \Delta f_{0}\left(t_{0}\right)
$$

and hence

$$
\mathcal{D} \Phi\left(t_{0}\right)=\alpha\left(\dot{f}_{1}\left(t_{0}\right), \Delta f_{0}\left(t_{0}\right), \Delta f_{1}\left(t_{0}\right)\right)
$$

Let us calculate $\mathcal{D}\left\langle\ddot{f}_{1}, \ddot{f}_{1}\right\rangle=2\left\langle\mathcal{D} \ddot{f}_{1}, \ddot{f}_{1}\right\rangle$. Decompose

$$
\ddot{f}_{1}=\frac{\left(\ddot{f}_{1}, \Delta f_{0}, \Delta f_{1}\right)}{\left(\dot{f}_{1}, \Delta f_{0}, \Delta f_{1}\right)} \dot{f}_{1}+\frac{\left(\dot{f}_{1}, \ddot{f}_{1}, \Delta f_{1}\right)}{\left(\dot{f}_{1}, \Delta f_{0}, \Delta f_{1}\right)} \Delta f_{0}+\frac{\left(\dot{f}_{1}, \Delta f_{0}, \ddot{f}_{1}\right)}{\left(\dot{f}_{1}, \Delta f_{0}, \Delta f_{1}\right)} \Delta f_{1} .
$$

Since

$$
\left\langle\mathcal{D} \ddot{f}_{1}\left(t_{0}\right), \dot{f}_{1}\left(t_{0}\right)\right\rangle=0, \quad \text { and } \quad\left\langle\mathcal{D} \ddot{f}_{1}\left(t_{0}\right), \Delta f_{1}\left(t_{0}\right)\right\rangle=0 \text {, }
$$

we get

$$
\mathcal{D}\left\langle\ddot{f}_{1}\left(t_{0}\right), \ddot{f}_{1}\left(t_{0}\right)\right\rangle=2 \frac{\left(\dot{f}_{1}\left(t_{0}\right), \ddot{f}_{1}\left(t_{0}\right), \Delta f_{1}\left(t_{0}\right)\right)}{\left(\dot{f}_{1}\left(t_{0}\right), \Delta f_{0}\left(t_{0}\right), \Delta f_{1}\left(t_{0}\right)\right)}\left\langle\mathcal{D} \ddot{f}_{1}\left(t_{0}\right), \Delta f_{0}\left(t_{0}\right)\right\rangle
$$

By Eq. (8) we have

$$
\left\langle\mathcal{D} \ddot{f}_{1}, \Delta f_{0}\right\rangle=-\left\langle\ddot{f}_{1}, \mathcal{D} \Delta f_{0}\right\rangle
$$

Hence after the substitution of $\mathcal{D} \Delta f_{0}\left(t_{0}\right)$ in the first summand one gets

$$
\left\langle\mathcal{D} \ddot{f}_{1}, \Delta f_{0}\right\rangle=\alpha\left(\dot{f}_{1}\left(t_{0}\right), \ddot{f}_{1}\left(t_{0}\right), \Delta f_{0}\left(t_{0}\right)\right)=\frac{\left(\dot{f}_{1}\left(t_{0}\right), \ddot{f}_{1}\left(t_{0}\right), \Delta f_{0}\left(t_{0}\right)\right)}{\left(\dot{f}_{1}\left(t_{0}\right), \Delta f_{0}\left(t_{0}\right), \Delta f_{1}\left(t_{0}\right)\right)} \mathcal{D} \Phi\left(t_{0}\right) .
$$

Therefore, we obtain

$$
\mathcal{D}\left\langle\ddot{f}_{1}\left(t_{0}\right), \ddot{f}_{1}\left(t_{0}\right)\right\rangle=2 \frac{\left(\dot{f}_{1}\left(t_{0}\right), \ddot{f}_{1}\left(t_{0}\right), \Delta f_{1}\left(t_{0}\right)\right)\left(\dot{f}_{1}\left(t_{0}\right), \ddot{f}_{1}\left(t_{0}\right), \Delta f_{0}\left(t_{0}\right)\right)}{\left(\dot{f}_{1}\left(t_{0}\right), \Delta f_{0}\left(t_{0}\right), \Delta f_{1}\left(t_{0}\right)\right)^{2}} \mathcal{D} \Phi\left(t_{0}\right) .
$$


Since the statement does not depend on the choice of the basis and invariant under isometries, we get the statement for all the points.

Let us show a formula of a discrete shift.

Corollary 3 (On discrete shift.) Let $f$ be a strongly regular 3-ribbon surface in $C^{1,2,2,1}\left([a, b], \mathbb{R}^{3}\right)$. Then the following holds:

$$
\mathcal{D} \Phi_{2}(t)=\frac{\left(\dot{f}_{1}(t), \ddot{f}_{1}(t), \Delta f_{0}(t)\right)}{\left(\dot{f}_{2}(t), \ddot{f}_{2}(t), \Delta f_{2}(t)\right)} \frac{\left(\dot{f}_{2}(t), \Delta f_{1}(t), \Delta f_{2}(t)\right)^{2}}{\left(\dot{f}_{1}(t), \Delta f_{0}(t), \Delta f_{1}(t)\right)^{2}} \mathcal{D} \Phi_{1}(t)
$$

Proof The statement follows directly from Propositions 11 and 12.

\subsection{Necessary Condition of Infinitesimal Flexibility}

In this subsection we write down the infinitesimal flexibility monodromy conditions for 3-ribbon surfaces (via continuous shifts of Proposition 10 and discrete shifts of Corollary 3). Recall that

$$
\Lambda(t)=\frac{\left(\dot{f}_{1}(t), \ddot{f}_{1}(t), \Delta f_{0}(t)\right)}{\left(\dot{f}_{2}(t), \ddot{f}_{2}(t), \Delta f_{2}(t)\right)} \frac{\left(\dot{f}_{2}(t), \Delta f_{1}(t), \Delta f_{2}(t)\right)^{2}}{\left(\dot{f}_{1}(t), \Delta f_{0}(t), \Delta f_{1}(t)\right)^{2}}
$$

and

$$
H_{i}(t)=\frac{\left(\dot{f}_{i}(t), \Delta \dot{f}_{i-1}(t), \Delta f_{i}(t)\right)+\left(\dot{f}_{i}(t), \Delta f_{i-1}(t), \Delta \dot{f}_{i}(t)\right)}{\left(\dot{f}_{i}(t), \Delta f_{i-1}(t), \Delta f_{i}(t)\right)}, \quad i=1,2
$$

Theorem 3 Let $f$ be a strongly regular 3 -ribbon surface in $C^{1,2,2,1}\left([a, b], \mathbb{R}^{3}\right)$. If the surface $f$ is infinitesimally flexible then for every $t_{1}, t_{2} \in[a, b]$ we have

$$
\Lambda\left(t_{2}\right) \cdot \exp \left(\int_{t_{1}}^{t_{2}} H_{1}(t) d t\right)=\Lambda\left(t_{1}\right) \cdot \exp \left(\int_{t_{1}}^{t_{2}} H_{2}(t) d t\right)
$$

Remark 10 The condition of the proposition can be written in the "almost" equivalent infinitesimal form:

$$
\dot{\Lambda}-\left(H_{2}-H_{1}\right) \Lambda=0
$$

Here the left hand side expression is considered as a function in the interval $[a, b]$. The last expression has one disadvantage, $\dot{\Lambda}$ involves the third derivatives of $f_{1}$ and $f_{2}$, while the expressions in proposition involve only up to the second derivatives. 
Proof Let $f$ be infinitesimally flexible and $\mathcal{D} f$ be its infinitesimal nonzero flexion. On the one side by Corollary 3 we get relations between $\mathcal{D} \Phi_{1}\left(t_{i}\right)$ and $\mathcal{D} \Phi_{2}\left(t_{i}\right)$ for $i=1,2$. On the other side, Proposition 10 relates $\mathcal{D} \Phi_{i}\left(t_{1}\right)$ and $\mathcal{D} \Phi_{i}\left(t_{2}\right)$ for $i=1,2$. These four relations define the monodromy condition for $\Phi_{i}$ that is the condition in the theorem, Therefore, it holds if a surface is infinitesimally flexible.

Remark 11 Let us write a more simple expressions for a surface $w$ defined as

$$
\begin{aligned}
& w_{0}=f_{1}-\frac{1}{\left(\dot{f}_{1}, \Delta f_{0}, \Delta f_{1}\right)} \Delta f_{0} \\
& w_{1}=f_{1} \\
& w_{2}=f_{2} \\
& w_{3}=f_{2}+\frac{1}{\left(\dot{f}_{2}, \Delta f_{1}, \Delta f_{2}\right)} \Delta f_{2} .
\end{aligned}
$$

As one can see, all rulings of $w$ (if non-vanished) are parallel to the corresponding rulings of $f$. In addition the middle strip of $f$ coincides with the middle strip of $w$.

Notice that

$$
\left(\dot{w}_{1}(t), \Delta w_{0}(t), \Delta w_{1}(t)\right)=1 \quad \text { and } \quad\left(\dot{w}_{2}(t), \Delta w_{1}(t), \Delta w_{2}(t)\right)=1
$$

for all arguments $t$. Hence we have:

$$
\begin{aligned}
\Lambda & =\frac{\left(\dot{w}_{1}, \ddot{w}_{1}, \Delta w_{0}\right)}{\left(\dot{w}_{2}, \ddot{w}_{2}, \Delta w_{2}\right)}, \\
H_{i} & =-\left(\ddot{w}_{i}, \Delta w_{i-1}, \Delta w_{i}\right), \quad i=1,2 .
\end{aligned}
$$

Note that this expression holds momentary.

We conclude this subsection with the following open problem.

Problem 1 Find a sufficient condition for infinitesimal/finite flexibility of semidiscrete and 3-ribbon surfaces.

\section{Flexibility of $\boldsymbol{n}$-Ribbon Surfaces}

In this section we study flexibility questions for general case of $n \geq 2$. We show that a strongly regular $n$-ribbon surface has at most one degree of finite and infinitesimal flexibility (Sect. 5.1). Further we study flexions of combined $n$-ribbon surfaces (Sect. 5.2). This allows us to prove that finite or infinitesimal flexibility of regular $n$-ribbon surfaces is identified by finite or infinitesimal flexibility of all its 3-ribbon subsurfaces (Sect. 5.3).

\subsection{At Most One Degree of Flexibility for Strongly Regular $n$-ribbon Surfaces}

In this subsection we prove that all nontrivial infinitesimal flexions of strongly regular $n$-ribbon surfaces are strongly isometrically nontrivial, and that such surfaces has at most one degree of infinitesimal flexibility. 
Let us start with a useful tool to work with isometrically nontrivial flexions.

Lemma 6 An infinitesimal flexion of a weakly regularn-ribbon surface $f$ in the space $C^{0,1,0}\left([a, b], \mathbb{R}^{3}\right)$ is isometrically nontrivial at a point $(t, i)$ (where $i \in[1, \ldots, n-1]$ ) if and only if

$$
\mathcal{D}\left\langle\Delta f_{i-1}(t), \Delta f_{i}(t)\right\rangle \neq 0
$$

Proof Since $f$ is weakly regular, the pairs of vectors $\left(\dot{f}_{i}, \Delta f_{i-1}\right)$ and $\left(\dot{f}_{i}, \Delta f_{i}\right)$ span two non-coinciding 2 -spaces $\pi_{1}$ and $\pi_{2}$.

Since $\pi_{1}$ and $\pi_{2}$ do not coincide, the condition $\mathcal{D}\left\langle\Delta f_{i-1}, \Delta f_{i}\right\rangle \neq 0$ is equivalent to the fact that the infinitesimal flexion of the angle between $\pi_{1}$ and $\pi_{2}$ is non-zero. Therefore, by Definition 10 the last is equivalent to $f$ being isometrically nontrivial at a point $(t, i)$.

In the next proposition we prove two important preliminary statements.

Proposition 13 Consider $n \geq 2$. Let $f$ be a strongly regular $n$-ribbon surface in the space $C_{0}^{1,2,2, \ldots, 2,1}\left([a, b], \mathbb{R}^{3}\right)$. Then the following two statements hold.

(i) Every isometrically nontrivial infinitesimal flexion of $f$ is strongly isometrically nontrivial (i.e., $f$ is isometrically nontrivial at every point $(t, i)$ ).

(ii) For every regular isometric deformation $\gamma$ there exists a locally monotone function $\xi$ such that $\gamma(\xi)$ is a normalized isometric deformation of $f$ in some neighborhood of 0 .

Proof We prove Theorem 13(i) by induction in $n$.

Base of induction. Case $n=2$. Let $\mathcal{D} f$ be a nontrivial infinitesimal flexion of a weakly regular 2-ribbon surface $f$ in $C_{0}^{1,2,1}\left([a, b], \mathbb{R}^{3}\right)$. Therefore, there exists $t_{0}$ such that $\mathcal{D} \Phi\left(t_{0}\right) \neq 0$. By Proposition $10, \mathcal{D} \Phi\left(t_{0}\right) \neq 0$ implies that $\mathcal{D} \Phi(t) \neq 0$ for every $t \in[a, b]$. Hence, by Lemma $6 f$ is isometrically nontrivial at each point $(t, 1)$. Therefore, $f$ is strongly isometrically nontrivial.

Case $n=3$. Let $\mathcal{D} f$ be a nontrivial infinitesimal flexion of a regular 3-ribbon surface $f$. Therefore, there exists a point $\left(t_{0}, i\right)$ such that $\mathcal{D} \Phi_{i}\left(t_{0}\right) \neq 0$. Without loss of generality we assume that $i=1$ (the case $i=2$ is similar).

By the above in case $n=2$ we have: $\mathcal{D} \Phi_{1}\left(t_{0}\right) \neq 0$ implies that $\mathcal{D} \Phi_{1}(t) \neq 0$ for every $t \in[a, b]$. By Corollary 3 (and the strongly regular condition for $f$ ), for every $t \in[a, b]$ the statement $\mathcal{D} \Phi_{1}(t) \neq 0$ implies that $\mathcal{D} \Phi_{2}(t) \neq 0$. Hence, by Lemma $6 f$ is isometrically nontrivial at each point $(t, 1)$ and $(t, 2)$. Therefore, by Definition 10 $f$ is strongly isometrically nontrivial.

Step of induction. Consider a strongly regular $n$-ribbon surface $f$ with $n \geq 4$. Denote

$$
f^{1}=\left(f_{0}, f_{1}, \ldots, f_{n-1}\right), \quad f^{2}=\left(f_{1}, \ldots, f_{n-1}, f_{n}\right) \text { and } f^{12}=\left(f_{1}, \ldots, f_{n-1}\right)
$$

Let $\mathcal{D} f$ be isometrically nontrivial flexion of $f$. Without loss of generality we assume that $\mathcal{D} f^{1}$ is isometrically nontrivial. Hence by the induction assumption $\mathcal{D} f^{1}$ is strongly isometrically nontrivial. Thus, $\mathcal{D} f^{12}$ is strongly isometrically nontrivial. 
Since $f^{12}$ is a $(n-2)$-ribbon (with $n \geq 4$ ), we have that $\mathcal{D} f^{2}$ is isometrically nontrivial. Then by the induction assumption $\mathcal{D} f^{2}$ is strongly isometrically nontrivial.

Since $n \geq 3$ and both $f^{1}$ and $f^{2}$ are strongly isometrically nontrivial, $f$ is strongly isometrically nontrivial as well. This concludes the proof of Proposition 13(i).

Let us prove Proposition 13(ii). Let $\left\{f^{\lambda}\right\}$ be a regular isometric deformation of $f$ with parameter $\lambda \in[-\Lambda, \Lambda]$. Since $f$ has a fixed initial position, we have:

$$
\begin{gathered}
\mathcal{D}_{f^{\lambda}} \dot{f}_{1}^{\lambda_{0}}(a)=0, \quad \mathcal{D}_{f^{\lambda}} \Delta f_{0}^{\lambda_{0}}(a)=0, \quad \text { and } \\
\mathcal{D}_{f^{\lambda}} \Delta f_{1}^{\lambda_{0}}(a)=\alpha(\lambda) \dot{f}_{1}^{\lambda_{0}}(a) \times \Delta f_{1}^{\lambda_{0}}(a)
\end{gathered}
$$

for every $\lambda_{0} \in[-\Lambda, \Lambda]$.

Since $\left\{f^{\lambda}\right\}$ is regular, $\mathcal{D}_{f^{\lambda}} f^{0} \neq 0$. Therefore, there exists $\varepsilon>0$ such that $\mathcal{D}_{f^{\lambda}} f^{\lambda_{0}} \neq 0$ for $\lambda_{0} \in[-\varepsilon, \varepsilon]$. From Proposition 13(i) it follows that for every $\lambda_{0} \in[-\varepsilon, \varepsilon]$ the flexion $\mathcal{D}_{f^{\lambda}} f^{\lambda_{0}}$ is strongly isometrically nontrivial. Hence

$$
\alpha(\lambda) \neq 0 \text { for } \lambda \in[-\varepsilon, \varepsilon]
$$

Therefore, $\alpha(\lambda)$ is either a positive function or a negative function on $[-\varepsilon, \varepsilon]$. Denote

$$
\varphi(\lambda)=\int_{0}^{\lambda} \alpha(\tau) d \tau
$$

The function $\varphi$ is monotonous on $[-\varepsilon, \varepsilon]$, and hence there exists an inverse function $\varphi^{-1}$ on that interval. Denote

$$
\xi= \begin{cases}\varphi^{-1}, & \text { if } \varphi \text { is increasing } \\ -\varphi^{-1}, & \text { if } \varphi \text { is decreasing }\end{cases}
$$

Choose positive $\hat{\varepsilon}$ such that $\xi$ is defined on $[-\hat{\varepsilon}, \hat{\varepsilon}]$. Then

$$
\alpha(\xi(\lambda))=1
$$

for all $\lambda \in[-\hat{\varepsilon}, \hat{\varepsilon}]$. Hence $\gamma \circ \xi$ is a normalized isometric deformation.

Now we study degrees of finite and infinitesimal flexibility.

Remark 12 To be consistent we mention the case of 2-ribbon surfaces. Let $f$ be a weakly regular 2-ribbon semidiscrete surface in the space $C_{0}^{1,2,1}\left([a, b], \mathbb{R}^{3}\right)$. Then the following two statements hold.

(i) The surface $f$ has one degree of infinitesimal flexibility (Theorem 1).

(ii) The surface $f$ has one degree of finite flexibility (Theorem 2).

Let us prove a similar statement for the case of $n \geq 3$.

Theorem 4 Consider $n \geq 3$. Let $f$ be a strongly regular $n$-ribbon surface in the space $C_{0}^{1,2,2, \ldots, 2,1}\left([a, b], \mathbb{R}^{3}\right)$. Then the following two statements hold. 
(i) The surface $f$ has at most one degree of infinitesimal flexibility (i.e., all infinitesimal isometrically nontrivial flexions are proportional).

(ii) The surface $f$ is either finitely regularly rigid or has one degree of finite flexibility.

Proof (i) Let us assume the converse. Suppose there are two non-proportional isometrically-nontrivial flexions $\mathcal{D}^{1} f$ and $\mathcal{D}^{2} f$. By Proposition 13(i) both flexions are isometrically nontrivial at $(a, 1)$ Hence there exists $\alpha$ such that the infinitesimal flexion

$$
\mathcal{D} f=\mathcal{D}^{1} f-\alpha \mathcal{D}^{2} f
$$

is isometrically trivial at $(a, 1)$. Since $\mathcal{D}^{1} f$ and $\mathcal{D}^{2} f$ are non-proportional, there exists a point $(t, i)$ at which the flexion $\mathcal{D} f$ is isometrically nontrivial. Hence by Proposition 13(i) the infinitesimal flexion $\mathcal{D} f$ is isometrically nontrivial at $(a, 1)$. We arrive at a contradiction.

(ii) By Theorem 4(i) all infinitesimal flexions are proportional. Hence $f$ has at most one degree of infinitesimal flexibility. If it is zero, then the $f$ is infinitesimally rigid and hence it is finitely regularly rigid.

Let $f$ has one degree of infinitesimal flexibility. If $f$ does not have regular isometric deformations then $f$ is finitely regularly rigid. If $f$ has a regular isometric deformation, then $f$ has a normalized isometric deformation. Let us show that there exists at most one normalized isometric deformation of $f$. Let $\left\{f^{\lambda}\right\}$ be a normalized isometric deformation of $f$. As before we denote

$$
\Phi_{i}^{\lambda}=\left\langle\Delta f_{i-1}^{\lambda}, \Delta f_{i}^{\lambda}\right\rangle
$$

Notice that for normalized isometric deformations we have:

$$
\mathcal{D}_{f^{\lambda}}\left(\Phi_{0}^{\lambda}(a)\right)=\left(\Delta f_{0}^{\lambda}(a), \dot{f}_{1}^{\lambda}(a), \Delta f_{1}^{\lambda}\right)
$$

Therefore

$$
\Phi_{0}^{\lambda}(a)=\int_{0}^{\lambda}\left(\Delta f_{0}^{\mu}(a), \dot{f}_{1}^{\mu}(a), \Delta f_{1}^{\mu}\right) d \mu .
$$

Hence $\Phi_{0}^{\lambda}(a)$ coincides for all normalized isometric deformation of $f$. Therefore, by Proposition 10 and Corollary 3 for every $(t, i)$ and every parameter $\lambda$ the value

$$
\Phi_{i}^{\lambda}(t)
$$

is the same for all normalized isometric deformations. Therefore, by Theorem 2 every restriction of an arbitrary normalized isometric deformation $\left\{f^{\lambda}\right\}$ to the deformation of a 2-ribbon subsurface of $f$ does not depend on the choice of the normalized isometric deformation $\left\{f^{\lambda}\right\}$. Hence, all normalized isometric deformations of $f$ coincide. Therefore, $f$ has one degree of finite flexibility. 
Remark 13 The strong regularity condition of Theorem 13 is essential. Let us illustrate this with a simple example of a 3-ribbon surfaces which is not strongly regular. Consider

$$
f_{0}(t)=(t, 1,0) ; \quad f_{1}(t)=(t, 0,0) ; \quad f_{2}(t)=(t, 0,1) ; \quad f_{3}(t)=(t, 1,1)
$$

This surface has two distinct isometric deformations:

$$
\begin{aligned}
& \text { (i) } f_{0}^{\alpha}(t)=(t, 1,0) ; \quad f_{1}^{\alpha}(t)=(t, 0,0) ; \quad f_{2}^{\alpha}(t)=(t, 0,1) ; \\
& \quad f_{3}^{\alpha}(t)=(t, \cos \alpha, 1+\sin \alpha) ; \\
& \begin{aligned}
(i i) f_{0}^{\beta}(t)=(t, 1,0) ; \quad f_{1}^{\beta}(t)=(t, 0,0) ; \\
f_{2}^{\beta}(t)=(t, \sin \beta, \cos \beta) ; \quad f_{3}^{\beta}(t)=\left(t, \sqrt{2} \sin \left(\beta+\frac{\pi}{4}\right), \sqrt{2} \cos \left(\beta+\frac{\pi}{4}\right)\right) ;
\end{aligned}
\end{aligned}
$$

The infinitesimal flexions defined by these isometric deformations are not proportional.

\subsection{Flexibility of Combined $\boldsymbol{n}$-Ribbon Surfaces}

Let us now study finite and infinitesimal flexions of combined strongly regular semidiscrete surfaces.

As above, for an arbitrary semidiscrete surface $f=\left(f_{0}, f_{1}, \ldots, f_{n}\right)$ we denote

$$
f^{1}=\left(f_{0}, f_{1}, \ldots, f_{n-1}\right), \quad f^{2}=\left(f_{1}, \ldots, f_{n-1}, f_{n}\right) \text { and } f^{12}=\left(f_{1}, \ldots, f_{n-1}\right)
$$

\subsubsection{Infinitesimal Case}

We start with the infinitesimal case.

Theorem 5 (Infinitesimal flexibility of combined semidiscrete surfaces) Let $n \geq 4$. Consider a strongly regular $n$-ribbon semidiscrete surface $f$ in $C_{0}^{1,2,2, \ldots, 2,1}\left([a, b], \mathbb{R}^{3}\right)$. Let surfaces $f^{1}$ and $f^{2}$ defined by (19) be infinitesimally flexible. Then $f$ is infinitesimally flexible and has precisely one degree of infinitesimal flexibility.

Proof Let $\mathcal{D}^{1} f^{1}$ and $\mathcal{D}^{2} f^{2}$ be isometrically nontrivial flexions of $f^{1}$ and $f^{2}$ respectively. Since $f^{1}$ and $f^{2}$ are strongly regular $(n-1)$-ribbon surfaces for $n \geq 4$, Theorem 13 can be applied. By Theorem 13(i) the surfaces $f^{1}$ and $f^{2}$ are strongly isometrically nontrivial. Hence by Theorem 13(i) in case $n>4$ or by Theorem 1 (see Remark 12 above) in case $n=4$ the induced flexions $\tilde{\mathcal{D}}^{1} f^{12}$ and $\tilde{\mathcal{D}}^{2} f^{12}$ are proportional, i.e, there exists $\alpha$ such that

$$
\mathcal{D}^{1} f^{12}=\alpha \mathcal{D}^{2} f^{12}
$$

Thus the surface $f$ has the combined infinitesimal flexion $\mathcal{D} f$ that induces $\mathcal{D}^{1} f^{1}$ and $\alpha \mathcal{D}^{2} f^{2}$. This flexion is infinitesimally nontrivial, since the induced ones are infinitesimally nontrivial. Hence $f$ has at least one degree of infinitesimal flexibility. 
On the other hand by Theorem 13(i) the surface $f$ has at most one degree of infinitesimal flexibility. Hence $f$ is infinitesimally flexible and has one degree of infinitesimal flexibility.

\subsubsection{Finite Case}

We start with the following general statement on reparametrisation of deformations.

Proposition 14 Let $f$ be a strongly regular $n$-ribbon surface $(n \geq 2)$ in the space $C^{1,2,2, \ldots, 2,1}\left([a, b], \mathbb{R}^{3}\right)$. Consider two regular isometric deformations $\gamma_{1}$ and $\gamma_{2}$ of $f$. Then there exists a monotonous function $\xi$ such that $\gamma_{1}(\lambda)=\gamma_{2}(\xi(\lambda))$ in some small neighborhood of 0 .

Proof By Proposition 13(ii) there exists monotonous functions $\xi_{1}$ and $\xi_{2}$ such that $\gamma_{1} \circ \xi_{1}$ and $\gamma_{2} \circ \xi_{2}$ are normalized isometric deformations of $f$. Hence by Theorem 4 in case $n \geq 3$ and Theorem 2 (see Remark 12 above) in case $n=2$ we have

$$
\gamma_{1} \circ \xi_{1}=\gamma_{2} \circ \xi_{2}
$$

in some neighborhood of 0 . Set $\xi=\xi_{2} \circ \xi_{1}^{-1}$. The function $\xi$ is the monotonous function such that $\gamma_{1}(\lambda)=\gamma_{2}(\xi(\lambda))$ in some small neighborhood of 0 .

Theorem 6 (Finite flexibility of combined semidiscrete surfaces) Let $n \geq 4$. Consider a strongly regular $n$-ribbon semidiscrete surface $f$ in $C_{0}^{1,2,2, \ldots, 2,1}\left([a, b], \mathbb{R}^{3}\right)$. Let surfaces $f^{1}$ and $f^{2}$ defined by (19) be finitely flexible. Then $f$ is finitely flexible and has one degree of finite flexibility.

Proof By Theorem 4(ii) the surfaces $f^{1}$ and $f^{2}$ have one degree of finite flexibility. Therefore, there exist unique normalized isometric deformations $\gamma_{1}$ and $\gamma_{2}$ of $f^{1}$ and $f^{2}$ respectively. They induce two deformations $\tilde{\gamma}_{1}$ and $\tilde{\gamma}_{2}$ of the $(n-2)$-ribbon surface $f^{12}$. By Proposition 14 , since $n-2 \geq 2$, these two deformations locally parameterize the same curve in $C_{0}^{1,2,2, \ldots, 2,1}\left([a, b], \mathbb{R}^{3}\right)$, i.e., in the segment $[-\varepsilon, \varepsilon]$ for some $\varepsilon>0$ there exists a locally increasing function $\xi$ such that $\tilde{\gamma}_{1}(\lambda)=\tilde{\gamma}_{2}(\xi(\lambda))$.

Now consider the deformation $\gamma$ of the surface $f$ inducing both isometric deformations $\gamma_{1}$ for $f_{1}$ and $\gamma_{2} \circ \xi$ for $f_{2}$ in the segment $[-\varepsilon, \varepsilon]$ for some $\varepsilon>0$. The deformation $\gamma$ is isometric, since the induced ones are isometric. In addition, $\gamma$ is normalized, since its restriction to $f^{1}$ is a normalized isometric deformation. Hence $f$ is finitely flexible (and not finitely regularly rigid). Therefore, by Theorem 13(ii) $f$ has one degree of finite flexibility.

Remark 14 Note that the statements of Theorems 5 and 6 are no longer true for the case $n=3$. On the one hand every infinitesimally flexible (and, therefore, finitely flexible) 3-ribbon surface should satisfy a condition of Theorem 3, and, as it is easy to see, not every strongly regular 3-ribbon surface satisfies it. Hence there are strongly regular 3-ribbon surfaces that are finitely regularly rigid. On the other hand every 2-ribbon subsurface is weakly regular and hence it is finitely (and, therefore, infinitesimally) flexible. These two statements together contradict to the version of the statement of Theorems 5 for the case $n=3$. 


\subsection{An $n$-Ribbon Surface and Its 3-Ribbon Subsurfaces}

Let us finally describe a relation between finite/infinitesimal flexibility of $n$-ribbon surfaces and finite/infinitesimal flexibility of all 3-ribbon subsurfaces contained in them.

Theorem 7 Let $n \geq 4$. Consider a strongly regular $n$-ribbon surface $f$ in the space $C_{0}^{1,2,2, \ldots, 2,1}\left([a, b], \mathbb{R}^{3}\right)$. Then $f$ is infinitesimally flexible (and has one degree of infinitesimal flexibility) if and only if every 3-ribbon surface contained in the surface is infinitesimally flexible.

Proof Let $f$ be infinitesimally flexible. Therefore, there exists an infinitesimal flexion $\mathcal{D} f$ that is isometrically nontrivial. Therefore, by Proposition 13(i) the flexion $\mathcal{D} f$ is strongly isometrically nontrivial. Hence all its 3-ribbon surfaces are isometrically nontrivially flexible.

Suppose now that all 3-ribbon subsurfaces in a strongly regular surface $f$ are infinitesimally flexible. We prove that all $k$-ribbon surfaces are infinitesimally flexible for $k=3,4, \ldots, n$ by induction in $k$.

Base of induction. The case $k=3$ is tautological.

Step of induction. The $k$-th statement follows from the $(k-1)$-th by Theorem 5 .

Hence $f$ is infinitesimally flexible. Therefore, by Theorem 4(i) $f$ has one degree of infinitesimal flexibility.

For the finite flexibility we have the following.

Theorem 8 Let $n \geq 4$. Consider a strongly regular $n$-ribbon surface $f$ in the space $C_{0}^{1,2,2, \ldots, 2,1}\left([a, b], \mathbb{R}^{3}\right)$. Then this surface is finitely flexible (and has one degree of flexibility) if and only if every 3-ribbon surface contained in the surface is finitely flexible.

Remark 15 We think of this theorem as of a semidiscrete analogue to the statement of the paper Bobenko et al. (2008) on conjugate nets and all $(3 \times 3)$-meshes that they contain. In this paper we do not study phenomena related to non-compactness and hence we restrict ourselves to the case of compact $n$-ribbon surfaces.

Proof Let $f$ be finitely flexible. Therefore there exists a regular isometric deformation $\gamma$ of $f$. Since $\gamma$ is regular we have $\mathcal{D}_{\gamma} f \neq 0$. Since every finite flexion is infinitesimal flexion we are in position to apply Proposition 13(i). We get that the flexion $\mathcal{D}_{\gamma} f$ is strongly isometrically nontrivial. Hence the induced isometric deformations of all 3-ribbon surfaces have corresponding nontrivial finite flexions. Therefore, all 3-ribbon surfaces contained in $f$ are finitely flexible.

Suppose that all 3-ribbon subsurfaces in a strongly regular surface $f$ are finitely flexible. Let us prove that every $k$-ribbon surface $f$ is finitely flexible for $k=3,4, \ldots, n$ by induction in $k$.

Base of induction. The case $k=3$ is tautological.

Step of induction. The $k$-th statement follows from the $(k-1)$-th by Theorem 6 .

Hence $f$ is finitely flexible. Therefore, by Theorem 4(ii) $f$ has one degree of finite flexibility. 


\section{Isometric Deformation of Developable Semidiscrete Surfaces}

Suppose that all ribbons of a semidiscrete surface are developable, i.e., the vectors $\dot{f}_{i}, \Delta f_{i}$, and $\dot{f}_{i+1}$ are linearly dependent. We call such semidiscrete surfaces developable. In this section we describe an additional property for flexions of developable semidiscrete surfaces. We start with 2-ribbon surfaces.

Recall that

$$
H_{1}=\frac{\left(\dot{f}_{1}, \Delta \dot{f}_{0}, \Delta f_{1}\right)+\left(\dot{f}_{1}, \Delta f_{0}, \Delta \dot{f}_{1}\right)}{\left(\dot{f}_{1}, \Delta f_{0}, \Delta f_{1}\right)}
$$

(as defined on page 31 ).

Proposition 15 Consider a developable weakly regular 2-ribbon semidiscrete surface $f$ in $C^{1,2,1}\left([a, b], \mathbb{R}^{3}\right)$. Let

$$
\dot{f}_{0}(t)=a(t) \dot{f}_{1}(t)+b(t) \Delta f_{0}(t) \quad \text { and } \quad \dot{f}_{2}(t)=c(t) \dot{f}_{1}(t)+d(t) \Delta f_{1}(t)
$$

Then we have

$$
H_{1}(t)=d(t)-b(t)
$$

Proof First, we have

$$
\begin{aligned}
\left(\dot{f}_{1}, \Delta \dot{f}_{0}, \Delta f_{1}\right) & =\left(\dot{f}_{1}, \dot{f}_{1}-\dot{f}_{0}, \Delta f_{1}\right)=-\left(\dot{f}_{1}, \dot{f}_{0}, \Delta f_{1}\right)=-\left(\dot{f}_{1}, a \dot{f}_{1}+b \Delta f_{0}, \Delta f_{1}\right) \\
& =-b\left(\dot{f}_{1}, \Delta f_{0}, \Delta f_{1}\right) .
\end{aligned}
$$

Secondly, in a similar way we get

$$
\left(\dot{f}_{1}, \Delta f_{0}, \Delta \dot{f}_{1}\right)=d\left(\dot{f}_{1}, \Delta f_{0}, \Delta f_{1}\right)
$$

Finally we have

$$
H_{1}=\frac{\left(\dot{f}_{1}, \Delta \dot{f}_{0}, \Delta f_{1}\right)+\left(\dot{f}_{1}, \Delta f_{0}, \Delta \dot{f}_{1}\right)}{\left(\dot{f}_{1}, \Delta f_{0}, \Delta f_{1}\right)}=d-b
$$

This concludes the proof.

This fact gives a surprising corollary concerning the flexion of a 2-ribbon developable surface. Denote by $\alpha(t)$ the angle between $\Delta f_{0}(t)$ and $\Delta f_{1}(t)$.

Corollary 4 Let $f$ be a weakly regular 2-ribbon developable surface in the space $C^{1,2,1}\left([a, b], \mathbb{R}^{3}\right)$. Consider its isometric deformation $\gamma$. Let us choose the parameter $\lambda$ of $\gamma$ such that $\cos \left(\alpha\left(t_{0}\right)\right)$ linearly depends on $\lambda$. Then for every $t \in[a, b]$ the value $\cos (\alpha(t))$ linearly depends on $\lambda$. 
Proof First of all, notice that

$$
\left|\Delta f_{0}\right|\left|\Delta f_{1}\right| \cos \alpha=\left\langle\Delta f_{0}, \Delta f_{1}\right\rangle=\Phi
$$

and hence

$$
\cos \alpha=\frac{\Phi}{\left|\Delta f_{0}\right|\left|\Delta f_{1}\right|}
$$

By Proposition 10 and further by Proposition 15 we have

$$
\mathcal{D}_{\gamma} \Phi\left(t_{1}\right)=\mathcal{D}_{\gamma} \Phi\left(t_{0}\right) \cdot \exp \left(\int_{t_{0}}^{t_{1}} H_{1}(t) d t\right)=\mathcal{D}_{\gamma} \Phi\left(t_{0}\right) \cdot \exp \left(\int_{t_{0}}^{t_{1}}(d(t)-b(t)) d t\right)
$$

Therefore, the ratio $\mathcal{D}_{\gamma} \Phi\left(t_{1}\right) / \mathcal{D}_{\gamma} \Phi\left(t_{0}\right)$ is a nonzero constant that depends entirely on the inner geometry of a 2 -ribbon surface, but not on its embedding in $\mathbb{R}^{3}$. Therefore, the ratio

$$
\frac{\cos \alpha\left(t_{1}\right)}{\cos \alpha\left(t_{0}\right)}=\frac{\mathcal{D}_{\gamma} \Phi\left(t_{1}\right)}{\mathcal{D}_{\gamma} \Phi\left(t_{0}\right)} \frac{\left|\Delta f_{0}\left(t_{0}\right)\right|\left|\Delta f_{1}\left(t_{0}\right)\right|}{\left|\Delta f_{0}\left(t_{1}\right)\right|\left|\Delta f_{1}\left(t_{1}\right)\right|}
$$

is a nonzero constant that depends entirely on the inner geometry of a 2-ribbon surface but not on its embedding in $\mathbb{R}^{3}$ as well. This implies the statement of the corollary.

In fact, Corollary 4 implies a similar statement for an isometric deformation of a strongly regular $n$-ribbon developable surface.

Corollary 5 Consider a strongly regular finitely flexible $n$-ribbon developable surface of $f$ in $C^{1,2,1}\left([a, b], \mathbb{R}^{3}\right)$. Let $\gamma$ be a nontrivial isometric deformation of $f$ (i.e., $\left.\mathcal{D}_{\gamma} f \neq 0\right)$. Then there exists a choice of the parameter $\lambda$ of the deformation $\gamma$, such that for all $t \in[a, b]$ and $i \in\{1, \ldots, n-1\}$ all the cosines of the corresponding angles $\alpha_{i}(t)$ linearly depend on $\lambda$ (here $\alpha_{i}(t)$ denotes the angle between $\Delta f_{i}(t)$ and $\left.\Delta f_{i+1}(t)\right)$.

Acknowledgments The author is grateful to J. Wallner for constant attention to this work, A. Weinmann for good remarks, and the unknown reviewer for excellent comments and suggestions. This work has been partially supported by the project "Computational Differential Geometry" (FWF Grant No. S09209).

\section{References}

Bianchi, L.: Sopra alcone nuove classi di superficie e di sistemi tripli ortogonali. Ann. Matem. 18, 301-358 (1890)

Bobenko, A.I., Hoffmann, T., Schief, W.K.: On the integrability of infinitesimal and finite deformations of polyhedral surfaces, discrete differential geometry. In: Bobenko, A.I., Schröder, P., Sullivan, J.M., Ziegler, G.M. (eds.) Series: Oberwolfach Seminars, vol. 38, pp. 67-93. Birkhäuser, Basel (2008)

Bobenko, A.I., Suris, Y.B.: Discrete Differential Geometry. Integrable Structure. Graduate Studies in Mathematics, vol. 98. American Mathematical Society, Providence (2008)

Cartan, H.: Calcul différentiel. Hermann, Paris (1967) 
Eisenhart, L.P.: A Treatise on the Differential Geometry of Curves and Surfaces. Dover, New York (1960)

Izmestiev, I.: Classification of Flexible Kokotsakis Polyhedra with Quadrangular Base (2014). arXiv: 1411.0289

Karpenkov, O.: On the flexibility of Kokotsakis meshes. Geom. Dedicata 147(1), 15-28 (2010)

Karpenkov, O., Wallner, J.: On offsets and curvatures for discrete and semidiscrete surfaces. Beitr. Algebra Geom. 55(1), 207-228 (2014)

Kokotsakis, A.: Über bewegliche Polyeder. Math. Ann. 107, 627-647 (1932)

Müller, Ch., Wallner, J.: Semi-discrete isothermic surfaces. Results Math. 63, 1395-1407 (2013)

Pottmann, H., Wallner, J.: Infinitesimally flexible meshes and discrete minimal surfaces. Monatshefte Math. 153, 347-365 (2008)

Pottmann, H., Wallner, J.: Computational Line Geometry. Mathematics and Visualization. Springer, Berlin (2001)

Sabitov, I.K.: Local theory of bendings of surfaces. In: Geometry, III, Encyclopaedia Math. Sci., vol. 48, pp. 179-256, Springer, Berlin (1992)

Stachel, H.: A kinematic approach to Kokotsakis meshes. Comput. Aided Geom. Des. 27(6), 428-437 (2010)

Wallner, J.: Semidiscrete surface representations. In: Bobenko, A., et al. (eds.) Discrete Differential Geometry, Oberwolfach Reports. 2009. Abstracts from the workshop held Jan 12-17 (2009)

Wallner, J.: On the semidiscrete differential geometry of A-surfaces and K-surfaces. J. Geom. 103, 161-176 (2012) 